\title{
Einführung von Kommunikationsstandards in Unternehmensnetzwerken - ein gestaltungsorientierter Ansatz bei dezentraler Entscheidungsstruktur
}

\author{
Bernd Heinrich, Mathias Klier, Marc-Andre Bewernik
}

Zusammenfassung: Aktuell diskutierte Standards und Technologien (bspw. im Bereich Web Services) werden die IT-gestützte Kommunikation zwischen Unternehmen stark verändern. Die Standardisierungsentscheidung geht jedoch mit dem Risiko einher, einen Standard einzuführen, den andere Unternehmen danach nicht verwenden. Somit entsteht durch die netzeffektbedingte Interdependenz der Standardisierungsentscheidungen ein Koordinationsproblem, das Standardisierungsproblem. Handelt es sich dabei um autonom entscheidende Akteure (bspw. eigenständige Unternehmen), denen die Standardisierungsentscheidungen der anderen Akteure im Netzwerk nicht bekannt sind, liegt das Standardisierungsproblem bei dezentraler Entscheidungsstruktur und unvollständiger Information vor. Die Akteure müssen folglich jeweils unter Unsicherheit über die eigene Einführung eines Standards entscheiden. Um daraus resultierende Ineffizienzen zu reduzieren und Fehlentscheidungen zu vermeiden, wird im Beitrag ein gestaltungsorientierter Ansatz entwickelt. Dieser soll die einzelnen Akteure im Netzwerk unterstützen, die Standardisierungsentscheidungen der anderen Akteure und somit die im Falle einer Standardisierung resultierenden Netzeffekte zu antizipieren. Kern des Ansatzes ist es dabei, die Interdependenz der Entscheidungen modellendogen zu repräsentieren und das resultierende Gleichungssystem zu lösen.

Schlüsselwörter: Kommunikationsstandards, Netzeffekte, Standardisierungsproblem, Entscheidungsunterstützung, Interdependentes Entscheidungsverhalten

\section{JEL-Classification: M15, O33}

Zitation: Einführung von Kommunikationsstandards in Unternehmensnetzwerken - ein gestaltungsorientierter Ansatz bei dezentraler Entscheidungsstruktur. in: ZEITSCHRIFT FÜR BETRIEBSWIRTSCHAFT (ZFB), Vol. 81, No. 4, 2011, pp. 447-485.

Prof. Dr. Bernd Heinrich, Institut für Wirtschaftsinformatik, Produktionswirtschaft und Logistik, Universität Innsbruck, Universitätsstraße 15, 6020 Innsbruck, Österreich.

E-Mail: Bernd.Heinrich@uibk.ac.at

Dr. Mathias Klier, Institut für Wirtschaftsinformatik, Produktionswirtschaft und Logistik, Universität Innsbruck, Universitätsstraße 15, 6020 Innsbruck, Österreich.

E-Mail: Mathias.Klier@uibk.ac.at

Dipl.-Inf. Marc-Andre Bewernik, FIM Kernkompetenzzentrum Finanz- \& Informationsmanagement - Lehrstuhl für BWL, Wirtschaftsinformatik, Informations- \& Finanzmanagement, Universität Augsburg, Universitätsstraße 12, 86159 Augsburg, Deutschland.

E-Mail: Marc.Bewernik@wiwi.uni-augsburg.de 


\section{Einleitung}

Kommunikations- und Technologiestandards nehmen in Zeiten stärkerer nationaler und internationaler Kooperationen von Unternehmen eine immer wichtigere Rolle ein. Dabei ist die Fragestellung, zu welchem Zeitpunkt man welchen Standard einführen soll, von großer Bedeutung. Nimmt man beispielhaft Web Service-Technologien, die aktuell intensiv diskutiert werden und versprechen, die IT-gestützte Prozessdurchführung in Wertschöpfungsnetzwerken nachhaltig zu verändern, so sind Unternehmen mit derartigen Fragestellungen konfrontiert. Hier gilt es zu analysieren, wann der richtige Zeitpunkt ist, einen neuen Web ServiceStandard einzuführen, um von bisherigen, ggf. proprietären Technologien zu wechseln. Zwar spielen auch technische Merkmale von Standards eine Rolle. Jedoch sind diese, wie bereits in der Literatur diskutiert, oftmals nicht ausschlaggebend für eine Standarddiffusion in einem Netzwerk von Unternehmen. Vielmehr sind die ökonomischen Folgen einer Standardeinführung abzuwägen. Ist ein Unternehmen bspw. bereit Vorreiter zu sein, um die Chance wahrzunehmen früh von den Potenzialen neuer Standards zu profitieren? Oder ist das Risiko zu groß, als frühzeitiger Adoptor auf einen Standard zu setzen, den andere Unternehmen später nicht einführen? In einem Netzwerk autonom entscheidender Unternehmen (Akteure), denen realistischerweise jeweils nur ein Teil der Nutzen- und Kostengrößen der anderen Akteure bekannt ist, kann es bei der Einführung von Standards zu Fehlentscheidungen und erheblichen Ineffizienzen kommen. Auch ist zu beachten, dass in solchen Netzwerken Mechanismen wie bspw. eine zentrale, koordinierende Instanz oftmals kaum durchsetzbar sind (vgl. z. B. Weitzel 2004). Deshalb wird im vorliegenden Beitrag untersucht, wie Akteure die Standardisierungsentscheidungen der anderen Akteure im Netzwerk und damit die im Falle einer Standardisierung entstehenden Netzeffekte antizipieren können, um damit die eigene Entscheidung zur Standardeinführung zu unterstützen. Konkret soll es für Unternehmen damit möglich werden, ineffiziente, dezentrale Standardisierungsentscheidungen zu verbessern.

Zur Bearbeitung dieser Fragestellung folgt der Beitrag einer normativen, quantitativen Modellierung (vgl. Bertrand/Fransoo 2002; Meredith et al. 1989), d. h. es wird ein gestaltungsorientierter Ansatz zur Antizipation dezentraler Standardisierungsentscheidungen vorgestellt. Dafür ist der Beitrag wie folgt aufgebaut: In Abschnitt 2 werden der Forschungsgegenstand konkretisiert und ein Überblick über bestehende Literatur gegeben. Um die Basis für die Entwicklung des eigenen Ansatzes nachvollziehbar und transparent darzustellen, diskutiert der Abschnitt 3 wesentliche Annahmen und Definitionen (axiomatisch-deduktive Vorgehensweise). Danach wird in Abschnitt 4 der gestaltungsorientierte Ansatz zur Unterstützung dezentraler Standardisierungsentscheidungen bei unvollständiger Information vorgestellt. Die zentrale Idee des Ansatzes ist, die für das Standardisierungsproblem charakteristische, netzeffektbedingte Interdependenz der Entscheidungen der Akteure modellendogen im Ansatz zu berücksichtigen. Aufgrund der an realen Problemstellungen angelehnten, feingranularen Modellierung des Ansatzes ist (analog zu einigen verwandten Ansätzen) die analytische Ermittlung einer geschlossenen Lösung nicht möglich (für eine detaillierte Begründung vgl. Abschnitt 5). Deshalb wird in Abschnitt 5 auf eine Simulationsstudie zurückgegriffen, deren Design auf Buxmann et al. (1999), Weitzel et al. (2003a), Weitzel (2004) und Weitzel et al. (2006) zurückgeht (zur generellen Verfahrensweise vgl. Bertrand/Fransoo 2002). Ziel ist es dabei zu analysieren, inwiefern der neue Ansatz im Rahmen des Forschungsdesigns zur Verbesserung ineffizienter, dezentraler Entscheidungen beiträgt. Abschnitt 6 umfasst die Interpretation der Analyseergebnisse und deren Implikationen, bevor der letzte Abschnitt den Beitrag zusammenfasst und kritisch würdigt. 


\section{Forschungsgegenstand und Literaturüberblick}

In Abschnitt 2.1 erfolgt zunächst eine Einordnung der Problemstellung in existierende Forschungsstränge zur Einführung bzw. Erzeugung von Standards. Danach werden in Abschnitt 2.2 bestehende Ansätze diskutiert.

\subsection{Grundsätzliche Einordnung der Problemstellung}

In der Literatur werden Fragestellungen zur Standardisierung aus Sicht der Anbieter vs. Nutzer von Standards und hinsichtlich der Erzeugung vs. Einführung von Standards betrachtet. Abb. 1 gibt einen Überblick über Forschungsstränge und ausgewählte Forschungsfragen.

\begin{tabular}{|c|c|c|}
\hline & Erzeugung des Standards & $\begin{array}{l}\text { Einführung des Standards } \\
\text { („Diffusion“) }\end{array}$ \\
\hline Anbieter & $\begin{array}{l}\text { „Standards Engineering“ } \\
\text { Fragen des Designs eines Standards bspw. } \\
\text { hinsichtlich der Kompatibilität mit anderen } \\
\text { Standards }\end{array}$ & $\begin{array}{l}\text { „Sponsored Networks“ } \\
\text { Fragen der Beeinflussung der Standardeinführung } \\
\text { durch den Anbieter mittels Marktmacht, } \\
\text { Preissetzung etc. }\end{array}$ \\
\hline Nutzer & $\begin{array}{l}\text { „User Participation“ } \\
\text { Fragen der Einbindung der Nutzer beim Design } \\
\text { eines Standards (z. B. in Gremien wie der Object } \\
\text { Management Group oder dem World Wide Web } \\
\text { Consortium) }\end{array}$ & $\begin{array}{l}\text { „Unsponsored Networks“ } \\
\text { Fragen des Netzwerkverhaltens und der Antizipation } \\
\text { von Standardisierungsentscheidungen anderer } \\
\text { potenzieller Nutzer }\end{array}$ \\
\hline
\end{tabular}

Abb. 1: Forschungsstränge und Forschungsfragen (angelehnt an Weitzel et al. 2006)

Neben Fragestellungen aus Anbietersicht, die z. B. Produkt-, Preis- und Kommunikationsstrategien für Standards betreffen, kommt ökonomischen Fragestellungen zur Einführung von Standards aus Nutzersicht eine besondere Bedeutung zu (vgl. auch Weitzel et al. 2006). Hier geht es darum, die ökonomische Vorteilhaftigkeit einer Standardisierung aus Nutzersicht zu analysieren. Da diese Vorteilhaftigkeit wesentlich von den Adoptionsentscheidungen der anderen Akteure im Netzwerk abhängt, spielen hier u. a. Fragestellungen zur Antizipation dieser Entscheidungen sowie zum Netzwerkverhalten eine wichtige Rolle. Abhängig von den verfügbaren Informationen sowie der Entscheidungsstruktur im Netzwerk lassen sich das Standardisierungsproblem aus Nutzersicht untergliedern und die hier fokussierte Problemstellung einordnen (vgl. Abb. 2).

\begin{tabular}{|c|c|c|}
\hline & Zentrale Entscheidungsstruktur & Dezentrale Entscheidungsstruktur \\
\hline $\begin{array}{l}\text { Vollständige } \\
\text { Information } \\
\text { (über Nutzen-, } \\
\text { Kostengrößen usw.) }\end{array}$ & $\begin{array}{l}\text { Zentrale, koordinierende Instanz entscheidet auf } \\
\text { Basis vollständiger Information über die } \\
\text { Standardisierung einzelner Akteure }\end{array}$ & $\begin{array}{c}\text { Jeder einzelne Akteur entscheidet autonom auf } \\
\text { Basis vollständiger Information über andere } \\
\text { Akteure }\end{array}$ \\
\hline $\begin{array}{l}\text { Unvollständige } \\
\text { Information } \\
\text { (über Nutzen-, } \\
\text { Kostengrößen usw.) }\end{array}$ & $\begin{array}{l}\text { Zentrale Instanz entscheidet auf Basis } \\
\text { unvollständiger Information über die } \\
\text { Standardisierung einzelner Akteure } \\
\text { (z. B. Konzernzentrale vs. Konzerntöchter) }\end{array}$ & $\begin{array}{c}\text { Jeder einzelne Akteur entscheidet autonom auf } \\
\text { Basis unvollständiger Information über andere } \\
\text { Akteure }\end{array}$ \\
\hline
\end{tabular}

Abb. 2: Einordnung der Problemstellung

Bei zentraler Entscheidungsstruktur und vollständiger Information sind die optimalen Standardisierungsentscheidungen für das gesamte Netzwerk unter Sicherheit ermittelbar und zumindest theoretisch ohne Weiteres realisierbar. Dagegen entscheiden die einzelnen Akteure bei dezentraler Entscheidungsstruktur und unvollständiger Information jeweils autonom und unter Unsicherheit (nur ein Teil der Nutzen- und Kostengrößen der anderen Akteure ist bekannt) über die Einführung eines Standards. Hieraus kann eine Ineffizienz in Form der soge- 
nannten Standardisierungslücke resultieren, d. h. eine Diskrepanz zwischen der Lösung bei zentraler Entscheidungsstruktur und vollständiger Information respektive dezentraler Entscheidungsstruktur und unvollständiger Information.

Im Folgenden wird das Standardisierungsproblem bei dezentraler Entscheidungsstruktur und unvollständiger Information fokussiert. Konkret wird die Forschungsfrage untersucht, wie einzelne Akteure im Netzwerk die Standardisierungsentscheidungen der anderen Akteure und damit die zu erwartenden Netzeffekte antizipieren können, um damit die eigene Entscheidung zur Standardeinführung zu unterstützen. Dadurch soll die Standardisierungslücke reduziert und es für Unternehmen möglich werden, ineffiziente, dezentrale Standardisierungsentscheidungen zu verbessern.

\subsection{Bestehende Ansätze}

Mit Fokus auf das Standardisierungsproblem bedienen sich zahlreiche, analytische Ansätze aus der Ökonomie (Besen/Farrell 1994; Farrell/Saloner 1985; Farrell/Saloner 1986; Farrell/Saloner 1988; Katz/Shapiro 1985; Liebowitz/Margolis 1995; Matutes/Regibeau 1988) „der Gleichgewichtsanalyse, um Phänomene wie z. B. das Start-up-Problem, Marktversagen, Instabilität (tippiness) und Pfadabhängigkeit zur Untersuchung von Märkten mit Netzeffekten zu erklären" (Wendt et al. 2000).

Im Hinblick auf das hier betrachtete Standardisierungsproblem bei dezentraler Entscheidungsstruktur ist insbesondere der Beitrag von Farrell und Saloner (1985) hervorzuheben, der sowohl den Fall vollständiger Information als auch den Fall unvollständiger Information untersucht. Für Letzteren entwickeln Farrell und Saloner (1985) ein analytisches Modell mit zwei Perioden und zwei Akteuren, die jeweils unvollständige Information darüber besitzen, wie affin der jeweils andere Akteur im Netzwerk hinsichtlich der Einführung eines Standards bzw. einer neuen Technologie ist. Folglich haben die Akteure unter Unsicherheit über die eigene Einführung des Standards zu entscheiden. Im Modell wird die jeweilige Affinität $i$ eines Akteurs einen Standard einzuführen, als Realisierung einer auf dem Intervall $[0 ; 1]$ gleichverteilten Zufallsvariable (a priori Verteilung) modelliert, wobei ein höher Wert von $i$ eine stärkere Affinität des Akteurs repräsentiert et vice versa. Der jeweilige Nutzen für einen Akteur als Folge seiner Standardisierungsentscheidung - Beibehaltung der ursprünglichen Technologie $X$ vs. Einführung des Standards $Y$ - wird dabei als Wert einer Funktion $B^{i}(m, k)$ repräsentiert. Sie ist abhängig von der Affinität $i$ des Akteurs, seiner gewählten Technologie $k \in\{X, Y\}$ sowie der Anzahl Akteure $m \in\{1,2\}$, die sich insgesamt für eine Technologie entscheiden. Zur formalen Ableitung allgemeiner Ergebnisse treffen und explizieren Farrell und Saloner (1985) Annahmen hinsichtlich der Nutzenfunktion $B^{i}(m, k)$ :

Hier wird angenommen, dass die Nutzenfunktionen beider Akteure $B^{i}(2, Y)$ und $B^{i}(1, Y)$ für den neuen Standard $Y$ jeweils stetig und streng monoton steigend in $i$ (mit $i \in[0 ; 1]$ ) sind. Zudem soll für beide betrachteten Technologien $k \in\{X, Y\}$ jeweils $B^{i}(2, k)>B^{i}(1, k)$ gelten, d. h. wenn beide Akteure die gleiche Technologie nutzen, dann kann der jeweilige Akteur einen echt größeren Nutzen realisieren, als wenn nur ein Akteur diese Technologie nutzt. Daneben wird vorausgesetzt, dass $B^{1}(1, Y)>0$ und $B^{0}(2, Y)<B^{0}(1, X)$ erfüllt sind sowie $B^{i}(2, Y)-B^{i}(1, X)$ monoton steigend in $i$ (mit $i \in[0 ; 1]$ ) ist.

Auf dieser Grundlage leiten Farrell und Saloner (1985) formal eine symmetrische Gleichgewichtsstrategie $\left(i^{*}, i^{\#}\right)$ mit $i^{*}, i^{\#} \in[0 ; 1]$ und $i^{*}>i^{\#}$ für die Akteure ab. Dabei stellen $i^{*}$ bzw. $i^{\#}$ Schwellenwerte für die Affinität der Akteure dar, den Standard $Y$ in der ersten Periode bzw. (in Abhängigkeit von der Entscheidung des anderen Akteurs in der ersten Periode) den Standard $Y$ erst in der zweiten Periode einzuführen. Konkret führt ein Akteur den Standard in der ersten Periode gemäß der Gleichgewichtsstrategie genau dann ein, wenn für dessen Affinität $i \geq i^{*}$ gilt (akteursspezifische Affinität ist größer oder gleich der Affinität der Gleichgewichtsstrategie für die erste Periode). Bei $i^{*}>i \geq i^{\#}$ (akteursspezifische Affinität ist kleiner als die Affinität der Gleichgewichtsstrategie für die erste aber größer oder gleich derjenigen für die zweite Periode) erfolgt in der zweiten Periode genau dann eine Standardisierung, wenn der 
jeweils andere Akteur in der ersten Periode bereits standardisiert hat. Falls dagegen $i<i^{\#}$ gilt, d. h. die akteursspezifische Affinität ist kleiner als die Affinität der Gleichgewichtsstrategie für die zweite Periode, wird grundsätzlich von einer Einführung des Standards abgesehen. Auf Basis der formal nachgewiesenen, symmetrischen Gleichgewichtsstrategie gelingt es Farrell und Saloner (1985) beim Standardisierungsproblem bei dezentraler Entscheidungsstruktur und unvollständiger Information allgemein auftretende Phänomene aufzuzeigen und zu erklären. In diesem Zusammenhang werden insbesondere auch Situationen identifiziert und diskutiert, in denen bspw. ein Akteur seine Entscheidung zu standardisieren ex post bereut, oder eine Einführung des Standards durch beide Akteure unterbleibt, obwohl dies für die Akteure oder das gesamte Netzwerk sinnvoll wäre. Auf diese Weise wird ein wesentlicher allgemeiner Beitrag zur Erklärung der im Zuge des Standardisierungsproblems bei dezentraler Entscheidungsstruktur auftretenden Ineffizienzen (diese werden in Abschnitt 3.4 noch detailliert diskutiert) geleistet. Zudem wird anhand einer Modellerweiterung aufgezeigt, dass nicht bindende Absprachen der Akteure vor dem Treffen der jeweiligen Standardisierungsentscheidung nur eingeschränkt zur Reduzierung dieser Ineffizienzen beitragen können.

Neben diesem für das Standardisierungsproblem bei dezentraler Entscheidungsstruktur und unvollständiger Information besonders relevanten Beitrag ist kurz auf ausgewählte, weitere Beiträge einzugehen. So werden in (Farrell/Saloner 1986) im Vergleich zu (Farrell/Saloner 1985) eigens auch die Auswirkungen einer installierten Basis, die bereits einen anderen Standard nutzt, sowie dynamische Effekte im Falle einer im Zeitverlauf sukzessiven Einführung eines neuen Standards im Netzwerk betrachtet. Ineffizienzen, die im Rahmen einer dynamischen Betrachtung auftreten (z. B. Einführung des Standards erfolgt zu spät), werden hier anhand eines Modells mit zwei Akteuren untersucht und erklärt. Dabei entscheiden die Akteure nicht mehr zwingend gleichzeitig, sondern haben jeweils zu bestimmten Zeitpunkten, die zufallsbedingt anhand einer Poisson-Verteilung ermittelt werden, wiederkehrend die Möglichkeit, den Standard einzuführen und dem ggf. vorher standardisierenden anderen Akteur in seiner Entscheidung zu folgen. Die hierbei auftretenden Phasen der Inkompatibilität verwendeter Standards haben definierte ökonomische Konsequenzen und beeinflussen damit auch die Standardisierungsentscheidungen der Akteure. In einem weiteren Beitrag von Farrell und Saloner (1988) liegt der Fokus nicht mehr darauf, grundlegende Ineffizienzen beim Standardisierungsproblem bei dezentraler Entscheidungsstruktur und unvollständiger Information zu identifizieren und zu erklären. Vielmehr werden für den Fall, dass die Akteure jeweils unterschiedliche Technologien bzw. Standards bevorzugen, mögliche Koordinationsmechanismen (bspw. Abstimmungen im Rahmen von Komitees) mit Hilfe von analytischen Modellen mit zwei Akteuren untersucht und verglichen. Auf derartige Koordinationsmechanismen wird in Abschnitt 3.4 im Zuge der Konkretisierung der Problemstellung des Beitrags zwar nochmals eingegangen. Sie stehen allerdings hier nicht im Fokus.

Neben den Beiträgen aus der Ökonomie finden sich mit (Buxmann 1996; Buxmann et al. 1999; Weitzel 2004; Weitzel et al. 2003b; Weitzel et al. 2006; Wendt et al. 2000) Beiträge, die primär ebenfalls das Standardisierungsproblem bei dezentraler Entscheidung und unvollständiger Information betrachten. Zentraler Gegenstand dieser Arbeiten ist $u$. a. die Untersuchung der Standarddiffusion aus Nutzersicht bei zentraler vs. dezentraler Entscheidungsstruktur. Hierzu werden ein ökonomischer Rahmen und ein formales Modell zur Untersuchung der Standardisierungsprobleme dargelegt. Auf dieser Grundlage werden verschiedene Einflussfaktoren, wie z. B. die Höhe der Standardisierungskosten und die Netzwerktopologie, im Hinblick auf das Auftreten und den Umfang der Standardisierungslücke analysiert. Zwar sind diese Beiträge damit ebenfalls primär erklärungsorientiert. Durch die dort vorgenommene feingranulare Modellierung können diese aber dennoch den Ausgangspunkt für die Entwicklung eines hier angestrebten gestaltungsorientierten Ansatzes in Abschnitt 4 bilden. Deshalb wird im folgenden Abschnitt 3 detailliert auf die dort vorgeschlagene Modellierung eingegangen. 


\section{Ausgangspunkt für die Entwicklung eines gestaltungsorientierten Ansatzes}

Zunächst werden die grundlegenden Modellannahmen der Arbeiten von Buxmann (1996), Buxmann et al. (1999) und Weitzel et al. (2006) zum Standardisierungsproblem herausgearbeitet und expliziert (vgl. Abschnitt 3.1). Danach wird das Standardisierungsproblem bei zentraler Entscheidung und vollständiger Information formal definiert (vgl. Abschnitt 3.2). Dies ist Voraussetzung, um nach der formalen Definition des Standardisierungsproblems bei dezentraler Entscheidungsstruktur und unvollständiger Information (vgl. Abschnitt 3.3) in Abschnitt 3.4 den Begriff der Standardisierungslücke ebenfalls exakt beschreiben und damit auftretende Ineffizienzen bei dezentralen Standardisierungsentscheidungen quantifizieren zu können. Abschließend erfolgt auf dieser Basis in Abschnitt 3.5 eine weitere Konkretisierung der fokussierten Problemstellung.

\subsection{Basisannahmen zur Modellierung des Standardisierungsproblems}

Die Kommunikationsbeziehungen zwischen Akteuren, die über die Einführung eines Standards entscheiden, lassen sich wie in (Buxmann et al. 1999) formal als gerichtetes Netzwerk darstellen, wobei die $M$ Akteure durch Knoten repräsentiert werden (vgl. Abb. 3).

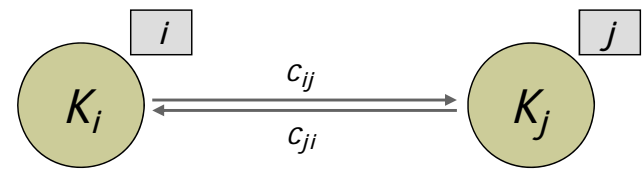

Abb. 3: Gerichtetes Netzwerk mit den Akteuren $i$ und $j$

Die gerichteten Kanten stellen den Informationsfluss zwischen den Akteuren dar und die Kantenbewertungen $c_{i j}$ bzw. $c_{j i}$ beschreiben die Informationskosten, die für Akteur $i$ bzw. $j$ aufgrund der Kommunikation über proprietäre Schnittstellen anfallen (vor Einführung des Standards). Um die Informationskosten zwischen zwei Akteuren einzusparen ${ }^{1}$, haben diese die Möglichkeit, ihre Kommunikation zu standardisieren. Dabei fallen für Akteur $i$ Standardisierungskosten in Höhe von $K_{i}$ an, die den einsparbaren Informationskosten in Höhe der $c_{i j}$ gegenüber zu stellen sind. Solche Einsparungen werden jedoch nur dann realisiert, wenn beide Akteure standardisieren. Die Basisannahmen der Modellierung des Standardisierungsproblems aus Nutzersicht sind in Anlehnung an (Buxmann 1996; Buxmann et al. 1999; Weitzel et al. 2006) wie folgt zu explizieren:

A.1 Das Netzwerk besteht aus $M \in \mathbb{N}$ Akteuren. Diese Anzahl ist jedem Akteur bekannt und hängt nicht davon ab, ob der Standard eingeführt wird oder nicht. Zunächst hat in der Ausgangssituation noch kein Akteur den Standard eingeführt ${ }^{2}$.

A.2 Zwischen allen Akteuren $i$ und $j(i, j \in\{1, \ldots, M\}, i \neq j)$ besteht eine wechselseitige Kommunikationsbeziehung ${ }^{3}$. Für die Kommunikation fallen für den sendenden Akteur $i$ die Informationskosten $c_{i j} \in \mathbb{R}^{+}$an (respektive die Kosten $c_{j i}$ für Akteur $j$ ). Die Kosten $c_{i j}$ spart Akteur $i$ nur dann ${ }^{4}$, falls beide Akteure $i$ und $j$ standardisieren $^{5}$. Ein Akteur $i$ kennt jeweils nur die Informationskosten $c_{i j}$ sowie $c_{j i}$ der direkten Kommunikationsbeziehungen zu anderen Akteuren $j$. Die Informationskosten zwischen dritten Akteuren sind Akteur $i$, da es sich bspw. um eigenständige Unternehmen handelt, (realistischerweise) nicht bekannt.

A.3 Standardisiert ein Akteur $i$, so fallen die Standardisierungskosten $K_{i} \in \mathbb{R}^{+}$an. Die Kosten $K_{i}$ sind allen Akteuren im Netzwerk bekannt.

Zur Begründung der Annahmen argumentieren Buxmann (1996), Buxmann et al. (1999) sowie Weitzel et al. (2006), dass sich die Anzahl der Akteure im Kommunikationsnetzwerk (vgl. Annahme A.1) sowie die jeweils zugehörige Höhe der Standardisierungskosten (vgl. Annahme A.3) für die einzelnen Akteure schnell und relativ verlässlich ermitteln lassen. Bei den Standardisierungskosten wird bspw. auf Marktpreise, Studien etc. verwiesen (vgl. z. B. auch Weitzel 2004 und die dort angeführten zahlreichen Fallstudien). Auch Annahme A.2 ist 
wenig einschränkend, da lediglich die Informationskosten der direkten Kommunikationsbeziehungen als bekannt angenommen werden. Die Informationskosten zwischen dritten Akteuren sind einem Akteur dagegen nicht bekannt ${ }^{6}$. Buxmann et al. (1999) begründen dies damit, dass diese Informationskosten für Unternehmen entweder zu ungenau zu schätzen sein dürften oder deren Beschaffung zu teuer sei. Die Informationskosten, an denen ein Akteur direkt beteiligt ist, sind dagegen für einen Entscheider in einem Unternehmen in Zusammenarbeit mit den eigenen Fach- und IT-Abteilungen durchaus ermittelbar. Hier weisen Weitzel et al. (2006) darauf hin, dass empirische Studien verdeutlichen, dass die notwendigen Informationen z. B. für Entscheider einfach zu ermitteln seien und diesen i. d. R. vorlägen. Zur konkreten Ermittlung der eingeführten Modellparameter und damit der Informationsbasis für den Fall der Einführung von X.500-Verzeichnisdiensten sei auf die detaillierte Darstellung in Weitzel et al. (2001) verwiesen.

\subsection{Problem bei zentraler Entscheidungsstruktur und vollständiger Information}

Im Folgenden wird zunächst kurz das Standardisierungsproblem bei zentraler Entscheidungsstruktur und vollständiger Information vorgestellt, um in Abschnitt 3.4 den Begriff der Standardisierungslücke formal definieren zu können. Dieses Standardisierungsproblem spiegelt bspw. eine Situation wider, in der die verschiedenen Abteilungen eines Unternehmens der Entscheidung einer zentralen, koordinierenden Instanz unterliegen, welche die Kostengrößen im Netzwerk vollständig kennt. In diesem Fall sind die obigen Basisannahmen A.1 bis A.3 durch folgende Annahme A. $4_{Z}$ (zentrale Entscheidung) zu ergänzen:

A. $4_{Z}$ Die Entscheidung über die Einführung des Standards bei den einzelnen Akteuren $i$ wird durch eine zentrale, koordinierende Instanz getroffen. Diese verfügt über vollständige Information hinsichtlich der Standardisierungs- und Informationskosten aller Akteure im Netzwerk. Die Standardisierung der einzelnen Akteure $i$ wird dabei so getroffen, dass dies zu minimalen Kommunikationskosten (Summe der Standardisierungs- und Informationskosten) für das gesamte Netzwerk führt.

Basierend auf A.1 bis A. $4_{Z}$ lassen sich die optimalen Standardisierungsentscheidungen mit Hilfe des folgenden linearen Programms ermitteln ${ }^{7}$ :

$$
\begin{array}{ll}
M I N & \sum_{i=1}^{M} K_{i} \cdot x_{i}+\sum_{i=1}^{M} \sum_{\substack{j=1 \\
j \neq i}}^{M} c_{i j} \cdot y_{i j} \\
\text { mit } & \forall i, j ; i \neq j \\
x_{i}+x_{j} \geq 2-2 \cdot y_{i j} & \forall i, j ; i \neq j \\
x_{i}, y_{i j} \in\{0 ; 1\} & \\
i, j \in\{1, \ldots, M\} &
\end{array}
$$

In der Zielfunktion werden die gesamten Kommunikationskosten minimiert, d. h. die Summe aus den Standardisierungskosten $K_{i}$ der einzelnen Akteure $i$ und den Informationskosten $c_{i j}$. Die Entscheidungsvariablen $x_{i} \in\{0 ; 1\}$ repräsentieren jeweils die Standardisierung des Akteurs $i$, d. h. $x_{i}=1$ bzw. $x_{i}=0$ bedeutet, Akteur $i$ wird standardisiert bzw. nicht standardisiert. Die Standardisierungskosten $K_{i}$ des Akteurs $i$ gehen somit nur für den Fall in die Zielfunktion ein, dass Akteur $i$ den Standard nutzt $\left(x_{i}=1\right)$, ansonsten ergibt sich $K_{i} \cdot x_{i}$ zu null. Die Binärvariablen $y_{i j}$ stellen sicher, dass die Informationskosten $c_{i j}$ nur entfallen, falls sowohl Akteur $i$ als auch Akteur $j$ den Standard nutzen (d. h. $y_{i j}=0$ ). Ansonsten ergibt sich $y_{i j}$ aufgrund der Nebenbedingung $x_{i}+x_{j} \geq 2-2 \cdot y_{i j}$ zu eins und die Informationskosten $c_{i j}$ fließen in die Zielfunktion ein. Zur Verdeutlichung des obigen Standardisierungsproblems erläutern wir hier das Beispiel eines Netzwerks mit sechs Akteuren (vgl. Abb. 4), das später weitergeführt wird. Es behandelt die Einführung von X.500-Verzeichnisdiensten, wobei die Informationsbasis an (Weitzel et al. 2001) angelehnt und für die Darstellung des Beispiels vereinfacht wurde. 
Verzeichnisdienste werden v. a. eingesetzt, um Daten in einem Unternehmen oder in Unternehmensnetzwerken zu sammeln, ,zentralisiert“ zu verwalten sowie diese Daten Benutzern und Applikationen wieder zur Verfügung zu stellen. Zentralisiert heißt dabei nicht, dass diese Daten nur an einer Stelle gespeichert und abrufbar sind, sondern vielmehr, dass diese konsistent und einheitlich im Netzwerk verwaltet werden (bspw. mittels Synchronisationsmechanismen). Dadurch wird ein qualitätsgerechter und standardisierter Zugriff erlaubt. Die X.500Verzeichnisdienste lehnen sich an die ISO/OSI-Standards an und werden derzeit häufig mit dem Lightweight Directory Access Protocol (LDAP) umgesetzt. Weitzel et al. (2001) untersuchen die Einführung von X.500-Verzeichnisdiensten in einem Netzwerk und erheben für sechs Institute eines Unternehmens die Standardisierungs- und Informationskosten.

Als Standardisierungskosten werden einmalige Produkt- und Einführungskosten sowie laufende Lizenz- und Administrationskosten für die Verzeichnisdienste betrachtet (letztere werden für einen definierten Zeithorizont barwertig berechnet). Interessant ist dabei, dass $u$. a. wegen der unterschiedlichen Größe der Institute die Standardisierungskosten durchaus stärker voneinander abweichen (vgl. Abb. 4). Bei den einsparbaren Informationskosten sind nach (Weitzel et al. 2001) ein geringerer Benutzeraufwand durch beschleunigte Suchvorgänge, ein verringerter Administrationsaufwand durch die Verzeichnisdienste und vermiedene Druckkosten durch den Wegfall gedruckter Adressverzeichnisse zu berücksichtigen. Auf dieser Basis erfolgen Kalkulationen zu den einsparbaren Kosten durch die Einführung der Verzeichnisdienste (aus Platzgründen wird dies hier nicht im Detail ausgeführt). Für die einzelnen Akteure (Institute) und den Fall der zentralen Entscheidung sind diese Größen in Abb. 4 dargestellt. Bspw. muss Akteur 1 bei Einführung der Verzeichnisdienste mit Standardisierungskosten von insgesamt 1.030.953 Geldeinheiten (GE) rechnen. Die einsparbaren Informationskosten in der Kommunikation mit Akteur 2 betragen 141.903 GE, falls dieser die Verzeichnisdienste ebenfalls einführt.

Wird nun über das gesamte Netzwerk hinweg - ohne Berücksichtigung der Vorteilhaftigkeit der Lösung aus Sicht der einzelnen Akteure - die Summe der anfallenden Kommunikationskosten minimiert (vgl. Zielfunktion (1)), so müssen alle Institute den Standard einführen. Hier stehen den Standardisierungskosten von insgesamt 2.248.924 GE einsparbare Informationskosten von 3.904.065 GE gegenüber, d. h die Einsparungen im Netzwerk sind mit insgesamt 1.655.141 GE maximal.

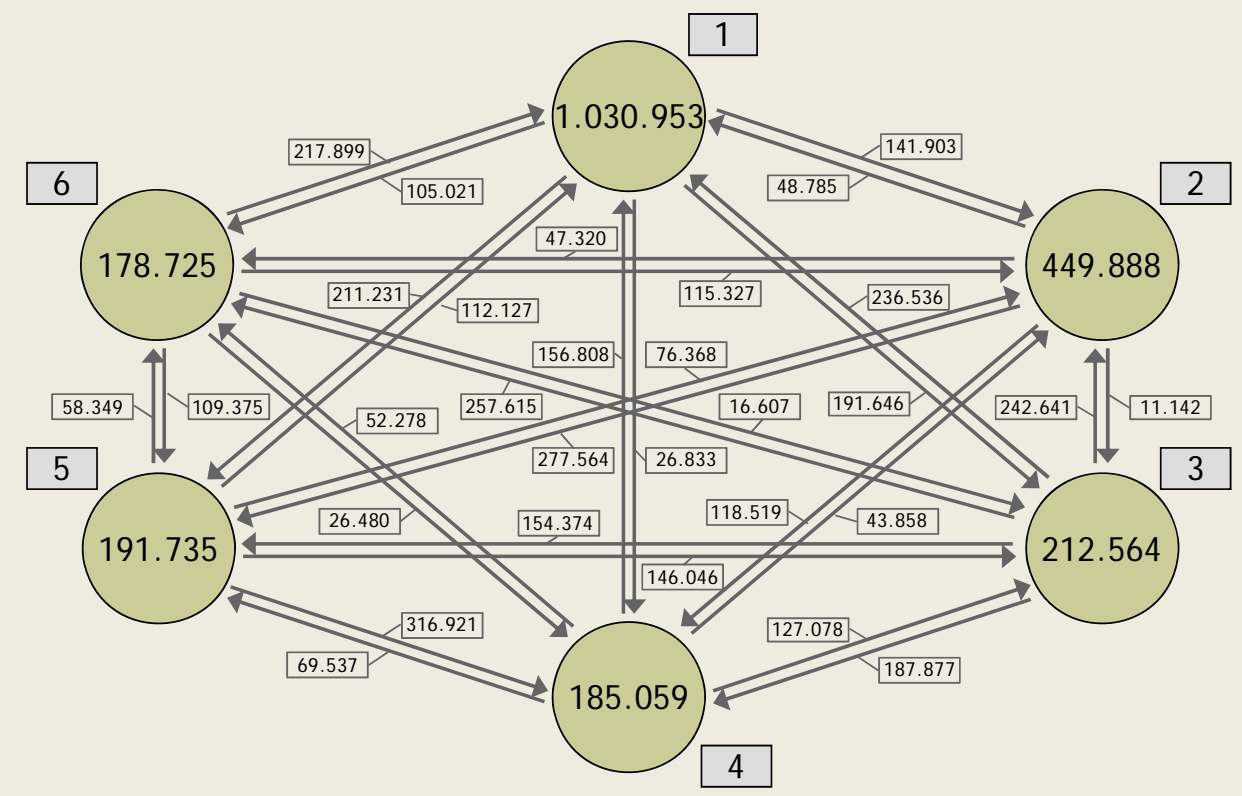

Abb. 4: Problem bei zentraler Entscheidungsstruktur und vollständiger Information

Dass die optimale Lösung für das Netzwerk nicht zwingend für jedes einzelne Institut vorteilhaft sein muss, lässt sich ebenfalls verdeutlichen. So resultieren bei Umsetzung der zentralen Lösung für Institut 1 zwar eine Informationskostenersparnis in Höhe von 676.634 GE jedoch 
auch Standardisierungskosten von 1.030.953 GE. Aus rein dezentraler Sicht des Instituts 1 wäre es demnach ökonomisch nicht sinnvoll zu standardisieren, obwohl die restlichen Institute von dieser Standardisierung profitieren.

\subsection{Problem bei dezentraler Entscheidungsstruktur und unvollständiger Information}

Handelt es sich bei den Akteuren dagegen um eigenständige Unternehmen, die autonom über die Einführung eines Standards entscheiden und denen realistischerweise jeweils nur ein Teil der Kostengrößen im Unternehmensnetzwerk bekannt ist, so liegt das hier fokussierte Standardisierungsproblem bei dezentraler Entscheidungsstruktur und unvollständiger Information vor. Auf Grundlage der obigen Basisannahmen A.1 bis A.3 lässt sich das Standardisierungsproblem am Beispiel von Akteur 2 wie in Abb. 5 dargestellt veranschaulichen. Hier wird insbesondere deutlich, dass Akteur 2 gemäß Annahme A. 2 zwar die Informationskosten $c_{2 j}$ sowie $c_{j 2}$ der direkten Kommunikationsbeziehungen zu anderen Akteuren $j$ kennt. Diese betragen bspw. für die Kommunikation mit Akteur 148.785 GE bzw. 141.903 GE. Dagegen sind Akteur 2 die Informationskosten zwischen dritten Akteuren, bspw. den Akteuren 1 und 6, nicht bekannt.

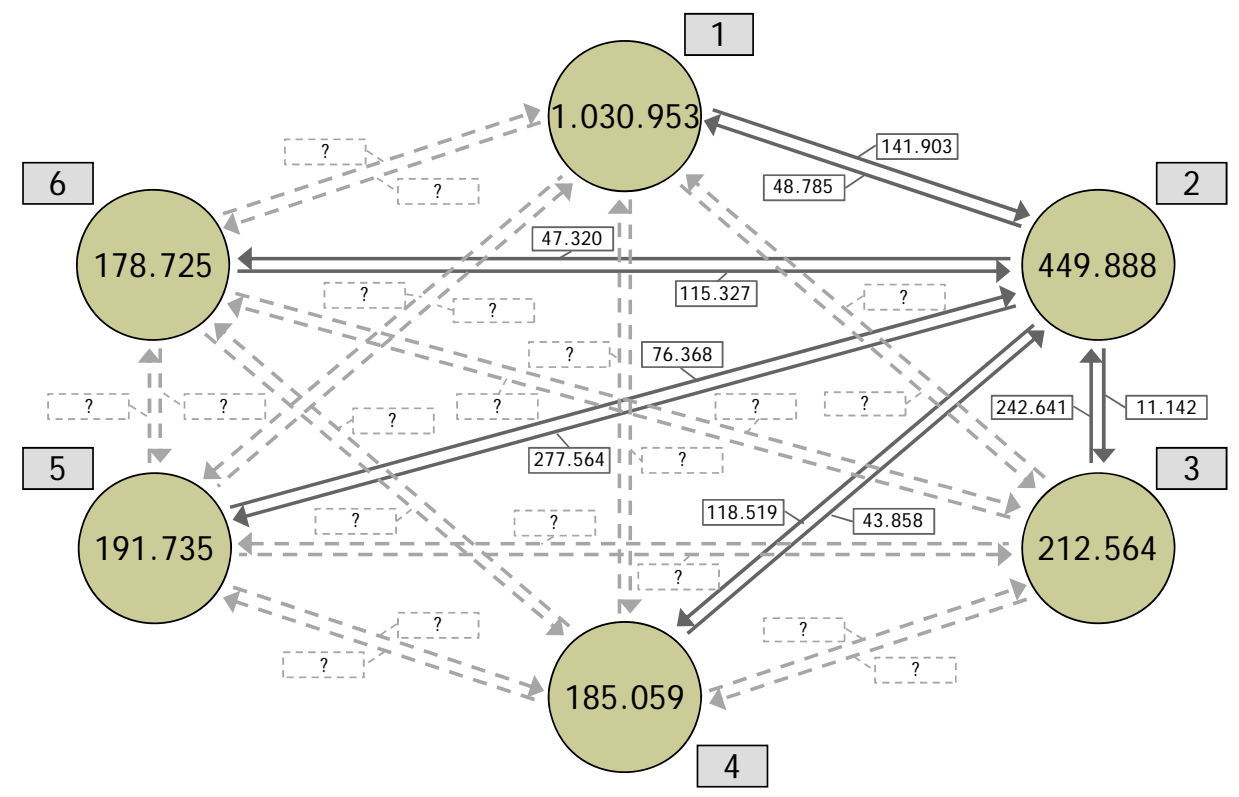

Abb. 5: Problem bei dezentraler Entscheidungsstruktur und unvollständiger Information

Bei der Modellierung des dezentralen Entscheidungsverhaltens verfahren bestehende Ansätze (vgl. z. B. Buxmann et al. 1999; Weitzel 2004; Weitzel et al. 2006) wie folgt:

Da einem Akteur $i$ - und dessen Sicht wird im Weiteren eingenommen - die Informationskosten $c_{j k}$ eines Akteurs $j$ mit $j, k \in\{1, \ldots, M\}, j \neq k$ und $j, k \neq i$ nicht bekannt sind, ist die Standardisierungsentscheidung des Akteurs $j$ unsicher. Insofern kann Akteur $i$ seine Standardisierungsentscheidung auch nicht unter Sicherheit treffen. Deshalb wird aus Sicht des Akteurs $i$ eine Wahrscheinlichkeit $p_{i j}$ dafür ermittelt, dass Akteur $j$ standardisiert. Mit Hilfe der Wahrscheinlichkeiten $p_{i j}$ entscheidet ein (risikoneutraler) Akteur $i$ dann auf Basis des Erwartungswerts $E\left(S_{i}\right)$ der einsparbaren Kommunikationskosten (vgl. Ausdruck (2)). Er standardisiert demnach genau dann, wenn dies für ihn einen positiven Erwartungswert $E\left(S_{i}\right)$ der einsparbaren Kommunikationskosten zur Folge hat. D. h. wenn die Differenz zwischen der Summe der erwarteten Einsparungen bei den Informationskosten $c_{i j}$ und den Standardisierungskosten $K_{i}$ positiv ist. Somit sind die Basisannahmen A.1 bis A.3 statt um Annahme A. $4_{Z}$ (zentrale Entscheidung) um die Annahme A.4 $4_{\mathrm{DZ}}$ (dezentrale Entscheidung) zu ergänzen:

A. $4_{\text {DZ }}$ Die Entscheidung über die Einführung des Standards trifft jeder Akteur $i$ autonom. Er standardisiert genau dann, wenn dies für ihn einen positiven Erwartungswert $E\left(S_{i}\right)$ der einsparbaren Kommunikationskosten zur Folge hat (Risikoneutralität). 
Das dezentrale Kalkül für Akteur $i$ ergibt sich damit zu:

$$
\begin{aligned}
& E\left(S_{i}\right)=\sum_{\substack{j=1 \\
j \neq i}}^{M} c_{i j} \cdot p_{i j}-K_{i} \stackrel{?}{\geq} 0 \\
& \begin{array}{c}
\text { Erwartete Einsparungen bei } \\
\text { den Informationskosten durch } \\
\text { die Standardisierung }
\end{array} \text { Standardisierungskosten } \\
& \text { mit } p_{i j} \in[0 ; 1] \forall j=1, \ldots, i-1, i+1, \ldots, M
\end{aligned}
$$

Bei der Modellierung des dezentralen Entscheidungsverhaltens kommt der Wahrscheinlichkeit $p_{i j}$ einer Standardisierung von Akteur $j$ aus Sicht des Akteurs $i$ eine zentrale Rolle zu. Bei ihrer Ermittlung liegt bei Buxmann et al. (1999) und Weitzel et al. (2006) folgende Annahme zugrunde:

A.5 Bur Ermittlung der Wahrscheinlichkeit $p_{i j}$ einer Standardisierung von Akteur $j$ (aus Sicht des Akteurs $i$ ) wird angenommen, dass alle $M$ Akteure, mit denen Akteur $j$ eine Kommunikationsbeziehung unterhält, mit Sicherheit standardisieren. D. h. Akteur $i$ nimmt die Wahrscheinlichkeiten $p_{j k}$ für alle $j, k \in\{1, \ldots, M\}$ und $j \neq k$ als eins an.

Daraus folgt für die Ermittlung der Wahrscheinlichkeiten unmittelbar, dass Akteur $j$ durch die Einführung des Standards alle Informationskosten $c_{j k}$ einsparen kann. Da zudem für Akteur $i$ die Informationskosten $c_{j k}$ des Akteurs $j$ zu allen anderen Akteuren $k \neq i$ unbekannt sind (vgl. Annahme A.2), wird hierzu in oben genannten Beiträgen Folgendes angenommen:

A. $6_{\mathrm{B}}$ Die für Akteur $i$ bekannten Informationskosten $c_{j i}$ sind repräsentativ für die unbekannten Informationskosten $c_{j k}$ des Akteurs $j$ mit allen anderen Akteuren $k(j, k \in\{1, \ldots, M\}$, $j \neq k$ und $j, k \neq i$ ).

Die Wahrscheinlichkeit $p_{i j}$ wird auf Basis der Annahmen A. $5_{\mathrm{B}}$ und A. $6_{\mathrm{B}}$ wie folgt definiert:

$$
p_{i j}=\frac{\sum_{\substack{k=1 \\ k \neq j}}^{M} c_{j k} \cdot p_{j k}-K_{j}}{\sum_{\substack{k=1 \\ k \neq j}}^{M} c_{j k} \cdot p_{j k}} \rightarrow \quad p_{i j}=\frac{c_{j i} \cdot(M-1)-K_{j}}{c_{j i} \cdot(M-1)}
$$

In Ausdruck (3) gibt der Zähler den Erwartungswert der einsparbaren Kommunikationskosten des Akteurs $j$ im Falle einer Standardisierung an. Der Nenner normiert die Wahrscheinlichkeit $p_{i j}$ auf den Wertebereich kleiner eins. Für den Fall, dass der Bruch einen Wert kleiner null annimmt - d. h. $c_{j i} \cdot(M-1)-K_{j}<0$ - gilt $p_{i j}=0$ (Knickstelle). Der Ausdruck (3) zur Ermittlung der Wahrscheinlichkeit wird danach zur Berechnung des Erwartungswerts $E\left(S_{i}\right)$ der einsparbaren Kommunikationskosten (vgl. Ausdruck (2)) berücksichtigt, um das dezentrale Entscheidungsverhalten eines Akteurs zu repräsentieren.

\subsection{Definition der Standardisierungslücke}

Mit Hilfe der dargestellten Modellierung können Ineffizienzen untersucht werden, die beim Standardisierungsproblem bei dezentraler Entscheidungsstruktur und unvollständiger Information im Vergleich zum Standardisierungsproblem bei zentraler Entscheidungsstruktur und vollständiger Information auftreten (vgl. hierzu auch Weitzel et al. 2006). So kann basierend auf der Ermittlung der Wahrscheinlichkeiten $p_{i j}$ analysiert werden, in welchen Entscheidungssituationen und in welchem Umfang Ineffizienzen, quantifiziert durch die Standardisierungslücke, auftreten. Auch kann geprüft werden, wie eine Veränderung der Entscheidungssituation (repräsentiert bspw. durch einen veränderten Parameterwert) den Umfang der Standardisie- 
rungslücke beeinflusst. Die Standardisierungslücke ist dabei als Differenz der eingesparten Kommunikationskosten im Netzwerk bei zentraler Entscheidung und vollständiger Information und den ex post tatsächlich eingesparten Kommunikationskosten bei dezentraler Entscheidung und unvollständiger Information definiert. Um die Standardisierungslücke zu veranschaulichen, wird das Beispiel der Einführung der X.500-Verzeichnisdienste weitergeführt:

Bei einer dezentralen Entscheidung unter unvollständiger Information sind zunächst für jeden einzelnen Akteur $i$ (hier Institut) die Wahrscheinlichkeiten $p_{i j}$ zu bilden. Betrachtet man beispielhaft Institut 2, so lassen sich nach dem Ansatz von Buxmann et al. (1999) und Weitzel et al. (2006) folgende Werte für die Wahrscheinlichkeiten $p_{2 j}$ ermitteln:

\begin{tabular}{|l|c|c|c|c|c|}
\hline & Institut 1 & Institut 3 & Institut 4 & Institut 5 & Institut 6 \\
\hline Wahrscheinlichkeit $p_{2_{j}}$ & 0 & 0,82 & 0,16 & 0,50 & 0,69 \\
\hline
\end{tabular}

Tab. 1: Ermittelte Standardisierungswahrscheinlichkeiten aus Sicht von Institut 2

Bei bekannten Informationskosten $c_{2 j}$ ergeben sich aus Sicht von Institut 2 erwartete Einsparungen in Höhe von 198.534 GE. Der Erwartungswert unter Berücksichtigung der Standardisierungskosten berechnet sich zu $E\left(S_{2}\right)=198.534 \mathrm{GE}-449.888 \mathrm{GE}=-251.354 \mathrm{GE}$. Demnach standardisiert Institut 2 bei eigener Entscheidungskompetenz nicht. Wenn sich die anderen Institute ebenfalls gemäß des Ansatzes von Buxmann et al. (1999) und Weitzel et al. (2006) verhalten, resultieren jeweils folgende erwartete Einsparungen $E\left(S_{i}\right)$ :

\begin{tabular}{|l|l|l|l|l|l|l|}
\hline & Institut 1 & Institut 2 & Institut 3 & Institut 4 & Institut 5 & Institut 6 \\
\hline $\begin{array}{l}\text { Erwartete } \\
\text { Einsparung } E\left(S_{i}\right)[\mathrm{GE}]\end{array}$ & -626.468 & -251.354 & 34.434 & -15.051 & 155.895 & -119.632 \\
\hline
\end{tabular}

Tab. 2: Erwartete Einsparungen $E\left(S_{i}\right)$ für die sechs Institute

Auf Basis des dezentralen Entscheidungsverhaltens führen folglich nur die Institute 3 und 5 den Standard ein.

Standardisieren aber lediglich diese beiden Institute, so kann Institut 3 bei Standardisierungskosten von 212.564 GE nur die Informationskosten zum Institut 5 in Höhe von 153.374 GE tatsächlich einsparen. Insofern ergeben sich ex post negative Einsparungen von -58.190 GE für Institut 3. Analog resultieren ex post für Institut 5 negative Einsparungen von $-45.689 \mathrm{GE}$ (= eingesparte Informationskosten zum Institut 3 von 146.046 GE abzüglich den Standardisierungskosten von $191.735 \mathrm{GE})$. Folglich ergibt sich bei dezentraler Entscheidung eine Summe der ex post tatsächlich eingesparten Kommunikationskosten von -103.879 GE.

Da bei einer zentralen Entscheidung eine Einsparung von 1.655.141 GE resultieren würde (s. o.), ergibt sich eine Standardisierungslücke von insgesamt 1.759.020 GE.

Auf Basis der bisherigen Ausführungen lassen sich die Ursachen für das Auftreten der Standardisierungslücke grundsätzlich unterscheiden (vgl. hierzu auch Farrell/Saloner 1985):

- Zum einen muss die Umsetzung der zentralen Lösung nicht für jeden Akteur vorteilhaft sein (vgl. Institut 1 in Abb. 4). Dies ist dann der Fall, wenn aus dezentraler Sicht die Standardisierungskosten nicht durch die eigene Kosteneinsparung bei zentraler Lösung gedeckt werden. Für den Akteur ergeben sich somit im Falle einer Standardisierung höhere Kommunikationskosten in Höhe von $\Delta a$. Wenn die übrigen Akteure im Netzwerk jedoch durch die Standardisierung insgesamt in Höhe von $\Delta b$ mit $\Delta b>\Delta a$ profitieren, ist diese aus Sicht des Netzwerkes ökonomisch vorteilhaft. Kommt jedoch kein (finanzieller) Ausgleich zwischen den Akteuren zustande, so wird - selbst bei vollständiger Information aller Akteure - die zentrale Lösung bei dezentraler Entscheidung nicht realisiert (Problem fehlender Kompensation). Theoretisch ist dieses Phänomen auf das Prinzip der individuel- 
len Rationalität nach Rapoport (2001) zurückzuführen. Demnach schließt sich ein Akteur keiner Koalition (hier Zusammenschluss aller Akteure) an, falls daraus finanzielle Nachteile im Vergleich zum isolierten Agieren resultieren.

- Zum anderen können Ineffizienzen auftreten, falls ein Akteur aus dezentraler Sicht nur dann standardisiert, wenn er sicher wäre, dass auch andere Akteure standardisieren. Erst dann übersteigen die einsparbaren Informationskosten die eigenen Standardisierungskosten. Abb. 6 verdeutlicht diese Situation am Beispiel der Einführung der X.500-Verzeichnisdienste: Links ist die dezentrale Lösung bei vollständiger Information (alle Institute außer Institut 1 würden standardisieren) und rechts die dezentrale Entscheidungssituation bei unvollständiger Information aus Sicht des Instituts 2 dargestellt. Wie die linke Graphik zeigt, lohnt sich für Institut 2 eine Standardisierung nur, falls auch die Institute 3, 4, 5 und 6 mit Sicherheit standardisieren. Für Institut 2 hätte dies einsparbare Kommunikationskosten von 4.657 GE zur Folge. Die Institute 3, 4, 5 und 6 würden bei der dezentralen Lösung und vollständiger Information in Höhe von 629.943 GE, 107.692 GE, 405.949 GE und 89.064 GE profitieren. Ohne die Informationskosten zwischen dritten Akteuren zu kennen, wird Institut 2 aber zunächst nicht standardisieren, sondern abwarten, ob andere Institute den Standard einführen. Tritt diese Situation auch bei anderen Instituten auf, so kommt es im Netzwerk zur wechselseitigen Blockade (,aggressives Abwarten“), dem so genannten Start-up-Problem (vgl. auch Farrell/Saloner 1985; Farrell/Saloner 1986). Im Gegensatz zum vorgenannten Problem unterbleibt hier die Einführung des Standards, obwohl dies aus Sicht jedes einzelnen der fünf Institute vorteilhaft wäre. Ursache hierfür ist die unvollständige Information.

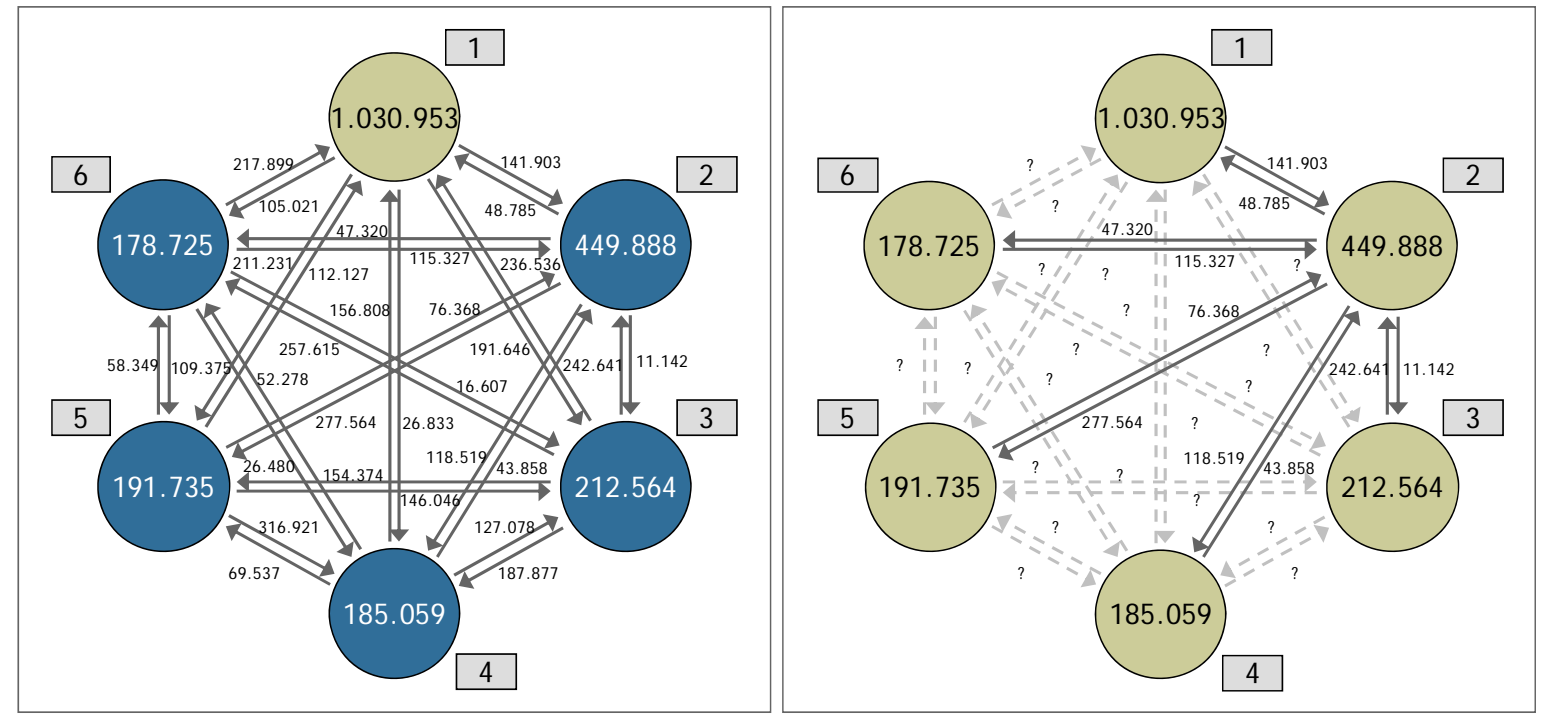

Abb. 6: Start-up-Problem bei dezentraler Entscheidung aus Sicht von Institut 2

\subsection{Konkretisierung der Problemstellung}

Um die Standardisierungslücke zu reduzieren und Ineffizienzen zu vermeiden, werden in der Literatur für das Standardisierungsproblem bei dezentraler Entscheidungsstruktur und unvollständiger Information u. a. folgende Koordinationsmechanismen diskutiert (vgl. z. B. Farrell 1987; Farrell/Saloner 1985; Farrell/Saloner 1988; Varian 1994; Weitzel 2003; Weitzel 2004):

[Einführung zentraler Koordinationsmechanismen] Hier wird die Einführung einer zentralen Instanz zur Koordination der dezentralen Entscheidungen vorgeschlagen, um das dezentrale Standardisierungsproblem bei unvollständiger Information (entspricht einem nichtkooperativen N-Personen-Spiel bei unvollständiger Information) in ein zentrales Entscheidungsproblem bei vollständiger Information zu überführen. Voraussetzung ist erstens, dass alle Akteure der zentralen, koordinierenden Instanz die Informationen über die eigene Kostenstruktur vollständig und richtig offenlegen. Insofern dürfen sich die Akteure nicht opportunistisch ver- 
halten, obwohl sie sich ggf. durch falsche Information Vorteile verschaffen können. Zweitens müssen sich alle Akteure an die Standardisierungsentscheidung dieser Instanz halten. Dies setzt hohe Anforderungen an deren Durchsetzungskraft. Zudem ist eine zentrale Koordination in vielen Fällen mit dem Prinzip der individuellen Rationalität nach Rapoport (2001) nur dann vereinbar, falls Ausgleichszahlungen zwischen den Akteuren festgelegt und auch durchgesetzt werden (vgl. die Ausführungen zum Problem fehlender Kompensation in Abschnitt 3.4). Insgesamt müsste daher eine große Vertrauensbasis sowohl gegenüber der zentralen, koordinierenden Instanz als auch gegenüber den anderen Akteuren bestehen, was insbesondere bei autonomen Unternehmen (vgl. das Beispiel in Weitzel 2004) sehr kritisch diskutiert wird. Zudem dürften die Einrichtung dieser Instanz, die Aufteilung der Kosten der Instanz (wer trägt diese Kosten zu welchen Anteilen?) und die Gewährleistung einer uneingeschränkten Kooperationsbereitschaft ebenfalls problematisch sein.

[Abstimmungen, Koalitionen und Kompensationszahlungen] Eine einfache Lösung, um die Standardisierungslücke zu reduzieren, scheinen Abstimmungen und verbindliche Absprachen (bspw. „runder Tisch“) zwischen den Akteuren zu sein. Hier ist eine Nähe zu den kooperativen N-Personen-Spielen gegeben, die sich u. a. schwerpunktmäßig mit Aspekten der Koalitionsbildung und charakteristischen Funktionen (welche Auszahlungen können einzelne Gruppen von Akteuren gemeinsam realisieren?) beschäftigt. Interessant ist dabei insbesondere, wie die gemeinsam in einer Koalition realisierbaren Einsparungen in einem zweiten Schritt zwischen den einzelnen Akteuren aufzuteilen sind. So weist Rapoport (2001) explizit darauf hin, dass selbst Lösungen, die gleichzeitig den Prinzipien der individuellen und kollektiven Rationalität genügen, ,grossly unfair“ erscheinen mögen. Zudem gestalten sich Abstimmungen zwischen den Akteuren ebenso problematisch, wenn sich aufgrund eines (potenziell) opportunistischen Verhaltens einzelner Akteure, sich ändernder Rahmenbedingungen oder wegen der zeitlichen Sequenzialität (vgl. hierzu ähnlich auch Farrell/Saloner 1986) dezentraler Standardisierungsentscheidungen die Ausgangsbasis ändert. Ähnliches gilt bei Kompensationszahlungen für Akteure, die aus individueller Sicht nicht standardisieren, dies jedoch im Rahmen einer Koalition tun sollen. Hier sind Fragen zu klären, wie: Welcher Akteur muss wie viel seiner Einsparungen weitergeben (dies könnte selbst dann zum Problem werden, falls es wie im obigen Beispiel für das Institut 2 zwar wegen der Einsparung von 4.657 GE vorteilhaft ist zu standardisieren, jedoch die Institute 3 und 6 weit mehr von einer Standardisierung des Instituts 2 profitieren, nämlich in Höhe von 242.641 GE bzw. 115.327 GE)? Welche Transparenz herrscht über die Einsparungen zwischen den Akteuren? Fordert ein Akteur nur Kompensationszahlungen zur Deckung seiner eigenen Kosten oder fordert er zusätzlich einen Anteil am Überschuss des Netzwerkes und wenn ja in welcher Höhe? In realen Netzwerken ist aufgrund derartiger Fragen eine Abstimmung zwischen den Akteuren oftmals schwierig (vgl. Weitzel et al. 2006). Daneben unterstreicht die Diskussion, dass es hier aus Sicht eines Akteurs durchaus vorteilhaft sein kann, Informationen über die eigene Kostenstruktur nicht richtig weiterzugeben und opportunistisch zu handeln. So können die eigene Position bei Verhandlungen verbessert, eigene Kompensationszahlungen reduziert oder höhere Kompensationszahlungen von anderen Akteuren gefordert werden.

Obige Koordinationsmechanismen werden somit durchaus kritisch gesehen. Deshalb wird im Folgenden untersucht, wie beim Standardisierungsproblem bei dezentraler Entscheidungsstruktur und unvollständiger Information auch ohne solche Koordinationsmechanismen zur Reduzierung der Standardisierungslücke und somit von Ineffizienzen beigetragen werden kann. Dazu gilt es für jeden Akteur die Standardisierungsentscheidungen der anderen Akteure im Netzwerk und damit die zu erwartenden Netzeffekte zu antizipieren. Jeder Akteur im Netzwerk entscheidet dabei autonom über die Einführung eines Standards. Informationen über die Kosten der Kommunikationsbeziehungen zwischen dritten Akteuren werden nicht bekannt gegeben (nichtkooperatives N-Personen-Spiel bei unvollständiger Information).

Hierzu soll ein gestaltungsorientierter Ansatz entwickelt werden, der zum einen der konkreten Entscheidungssituation Rechnung trägt, in der sich Unternehmen bei der Einführung von Standards befinden. Die in den Abschnitten 3.1 bis 3.3 vorgestellte Modellierung bildet in 
dieser Hinsicht einen geeigneten Ausgangspunkt. Zum anderen soll der gestaltungsorientierte Ansatz auch die Interdependenz der Standardisierungsentscheidungen modellendogen berücksichtigen und dem spieltheoretischen Charakter der Problemstellung gerecht werden.

\section{Gestaltungsorientierter Ansatz zur Antizipation dezentraler Standardisierungsent- scheidungen}

Im Weiteren wird ein Ansatz vorgestellt, der Akteure bei dezentraler Entscheidungsstruktur und unvollständiger Information unterstützen soll, die Standardisierungsentscheidungen anderer Akteure im Netzwerk und damit die zu erwartenden Netzeffekte zu antizipieren. Den Ausgangspunkt für die Entwicklung des Ansatzes bilden die oben explizierten Annahmen A.1 bis A. $4_{\text {DZ }}$ (vgl. Abschnitte 3.1 und 3.3). Die Annahmen A.5 $5_{B}$ und A. $6_{B}$, die das konkrete, dezentrale Entscheidungsverhalten nach Buxmann et al. (1999) und Weitzel et al. (2006) widerspiegeln (vgl. Abschnitt 3.3), werden im Folgenden ersetzt.

Gemäß Annahme A.4 $4_{\mathrm{DZ}}$ trifft ein Akteur $i$ die Entscheidung über die Einführung eines Standards auf Basis des Erwartungswerts $E\left(S_{i}\right)$ der einsparbaren Kommunikationskosten. Somit kommt der Ermittlung der Standardisierungswahrscheinlichkeiten $p_{i j}$ für die Akteure $j$ eine zentrale Rolle zu (vgl. Ausdruck (2)). Dabei sollen die Interdependenzen der Standardisierungsentscheidungen der Akteure sowie die entstehenden Netzeffekte als wesentliche Charakteristika modellendogen repräsentiert werden. Hierzu sind aus Sicht eines Akteurs $i$ die anderen Akteure entscheidungstheoretisch nicht als (starre) Umweltzustände zu modellieren. Sie stellen vielmehr Spieler mit eigenem Kalkül dar, welche die ihnen jeweils bekannten Informationen berücksichtigen und versuchen, wiederum Standardisierungswahrscheinlichkeiten für alle übrigen Akteure abzuleiten. Auf dieser Basis wählt jeder Akteur (Spieler) die wechselseitig beste Strategie.

Die Idee des gestaltungsorientierten Ansatzes ist demnach, zur Ermittlung der Standardisierungswahrscheinlichkeit $p_{i j}$ eines Akteurs $j$ (aus Sicht des Akteurs $i$ ) die Wahrscheinlichkeiten $p_{j k}$ des Akteurs $j$ für die anderen Akteure $k$ jeweils zu berücksichtigen. Für die Ermittlung dieser Wahrscheinlichkeiten $p_{j k}$ werden jedoch die Informationskosten $c_{j k}$ benötigt, die dem Akteur $i$ nicht bekannt sind (vgl. Annahme A.2). Deshalb sollen die für Akteur $i$ ermittelbaren Wahrscheinlichkeiten $p_{i k}$ anstelle der unbekannten Wahrscheinlichkeiten $p_{j k}$ Verwendung finden, da die Wahrscheinlichkeiten $p_{i k}$ bei unvollständiger Information jeweils repräsentieren, inwiefern die anderen Akteure $k$ standardisieren. Dies lässt sich mittels folgender, im Vergleich zu A.5 $5_{\mathrm{B}}$ modifizierter Annahme für das dezentrale Entscheidungsverhalten explizieren:

A.5* Bei der Ermittlung der Wahrscheinlichkeit $p_{i j}$ einer Standardisierung von Akteur $j$ (aus Sicht des Akteurs $i$ ) ist die Wahrscheinlichkeit $p_{i k}$ für alle $j, k \in\{1, \ldots, M\}$ und $j \neq k$ repräsentativ für die (unbekannten) Wahrscheinlichkeiten $p_{j k}$.

Legt man Annahme A.5* zugrunde, lässt sich aus Sicht von Akteur $i$ der Erwartungswert $E\left(S_{j}\right)$ der einsparbaren Kommunikationskosten des Akteurs $j$ im Falle einer Standardisierung wie folgt ermitteln:

$$
E\left(S_{j}\right)=\sum_{\substack{k=1 \\ k \neq j}}^{M} c_{j k} \cdot p_{j k}-K_{j} \quad \stackrel{\left(A .5^{*}\right)}{\Rightarrow} \quad E\left(S_{j}\right)=\sum_{\substack{k=1 \\ k \neq j}}^{M} c_{j k} \cdot p_{i k}-K_{j}
$$

Weiter ist zu spezifizieren, wie der Akteur $i$ die notwendigen Standardisierungswahrscheinlichkeiten $p_{i k}$ der Akteure $k$ konkret bestimmen kann. Diese Wahrscheinlichkeiten sollen als Funktion des Erwartungswerts $E\left(S_{k}\right)$ der einsparbaren Kommunikationskosten des Akteurs $k$ im Falle einer Standardisierung determiniert werden. Allgemein ist dazu eine Abbildung notwendig, die $p_{i k} \in[0 ; 1]$ mit $\partial p_{i k} / \partial \mathrm{E}\left(S_{k}\right)>0$ und $E\left(S_{k}\right) \in \mathbb{R}$ gewährleistet. Letzteres fordert für zunehmende Erwartungswerte $E\left(S_{j}\right)$ streng monoton steigende Wahrscheinlichkeiten $p_{i j}$ (Monotonieeigenschaft). Eine stetige Funktion, welche die gewünschten Abbildungseigenschaften 
beispielhaft besitzt, ist die Arcustangens-Funktion ArcTan[x] (in Anhang 1 wird die Verwendung dieses Funktionstyps formal begründet).

In das Argument $x$ geht dabei der Erwartungswert $E\left(S_{j}\right)$ der einsparbaren Kommunikationskosten ein (Ausdruck (4)). Jedoch ist hier zu beachten, dass bspw. ein Erwartungswert $E\left(S_{j}\right)$ von $1.000 \mathrm{GE}$ bei Standardisierungskosten $K_{j}$ von nur $100 \mathrm{GE}$ eine höhere Wahrscheinlichkeit der Standardisierung des Akteurs $j$ nach sich ziehen sollte als bei Standardisierungskosten von 100.000 GE. Deswegen wird der Erwartungswert zu den Standardisierungskosten $K_{j}$ ins Verhältnis gesetzt, d. h. der Erwartungswert wird durch die Standardisierungskosten dividiert:

$$
p_{i j}=f\left(\operatorname{ArcTan}\left[\frac{E\left(S_{j}\right)}{K_{j}}\right]\right)
$$

Die Arcutangens-Funktion bildet allgemein das Argument im Definitionsbereich $\mathbb{R}$ auf den Wertebereich $\left(-\frac{\pi}{2} ; \frac{\pi}{2}\right)$ ab. Dies stellt jedoch kein Problem dar, da trivialerweise durch Stauchung und Verschiebung der Arcustangens-Funktion eine Abbildung der Form $p_{i j}=f\left(\operatorname{ArcTan}\left[\frac{E\left(S_{j}\right)}{K_{j}}\right]\right) \rightarrow(0 ; 1)$ erreicht werden kann.

Anhand von Ausdruck (4) wird zudem deutlich, dass zur Berechnung des Erwartungswerts $E\left(S_{j}\right) \forall j \in\{1, \ldots, M\}$ mit $j \neq i$ ebenso die für Akteur $i$ unbekannten Informationskosten $c_{j k}$ zu antizipieren sind. Problematisch wäre es wie in Annahme A. $6_{\mathrm{B}} \mathrm{zu}$ unterstellen, dass dafür die bekannten Informationskosten $c_{j i}$ repräsentativ sind. Gründe hierfür sind, dass die Informationskosten $c_{j k}$ nicht nur von der verwendeten Technologie des Akteurs $j$, sondern auch von derjenigen des benachbarten Akteurs $k$ abhängig sein können oder dass der Akteur $j$ zur bisherigen Kommunikation jeweils eine eigene proprietäre Technologie zu jedem benachbarten Akteur eingesetzt hat. Dies stellt die Übertragbarkeit der Informationskosten $c_{j i}$ zwar nicht grundsätzlich in Frage, jedoch kann es bei einem Ausreißer bei den bekannten Informationskosten $c_{j i} \mathrm{zu}$ erheblichen Verzerrungen bei der Antizipation der unbekannten Informationskosten $c_{j k}$ kommen (Antizipation auf Basis eines Einzelwertes). Deshalb wird statt Annahme A. $6_{\mathrm{B}}$ folgende Annahme A. $6^{*}$ getroffen:

A.6* Für die dem Akteur $i$ unbekannten Informationskosten $c_{j k}$ des Akteurs $j$ mit allen anderen Akteuren $k(j, k \in\{1, \ldots, M\}, j \neq k$ und $j, k \neq i)$ sind sowohl die ihm bekannten Informationskosten $c_{j i}$ als auch die ihm bekannten Informationskosten $c_{k i}$ bestimmend.

Darauf basierend werden die Informationskosten $c_{j k}$ mit Hilfe des arithmetischen Mittels und der Standardabweichung der Werte $c_{k i}$ mit $k \in\{1, \ldots, M\}$ und $k \neq i$ ermittelt. Folgender Ausdruck $\tilde{c}_{j k}$ berücksichtigt die Informationskosten $c_{k i}$ in Form des arithmetischen Mittels $\bar{c}$ (mit

$\left.\bar{c}=\frac{\sum_{\substack{k=1 \\ k \neq i}}^{M} c_{k i}}{M-1}\right)$ sowie der Standardabweichung $\sigma\left(\right.$ mit $\sigma=\sqrt{\left.\frac{\sum_{\substack{k=1 \\ k \neq i}}^{M}\left(c_{k i}-\bar{c}\right)^{2}}{M-2}\right) \text { : }}$

$$
\widetilde{c}_{j k}=c_{j i}-\frac{c_{A B S}-\sigma}{c_{A B S}} \cdot\left(c_{j i}-\bar{c}\right) \text { mit } c_{A B S}>0
$$

Wie Ausdruck (6) zeigt, werden die Informationskosten $c_{j i}$ noch um einen Term korrigiert. Dieser berücksichtigt die Abweichung der Kosten $c_{j i}$ vom arithmetischen Mittel $\bar{c}$ sowie das Verhältnis der Standardabweichung $\sigma$ zur maximalen absoluten Abweichung $c_{A B S}$ der Informationskosten $c_{j i}$ vom arithmetischen Mittel $\bar{c}$ (mit $c_{A B S}=\operatorname{Max}\left[\operatorname{Abs}\left(c_{k i}-\bar{c}\right)\right]$ ). Der Vorteil dieser Ermittlung liegt in der Berücksichtigung zweier Effekte. Zum einen werden größere Ausreißer der Informationskosten $c_{j i}$ vom arithmetischen Mittel $\bar{c}$ zwar geglättet, jedoch in der Betrachtung nicht eliminiert. Zum zweiten wird auch eine starke bzw. geringe Streuung der Informationskosten $c_{j i}$ (Standardabweichung $\sigma$ ) berücksichtigt, da dies Ausdruck einer sehr 
heterogenen bzw. homogenen Kostenstruktur der Akteure ist. D. h. die Informationskosten werden als äußerst charakteristisch für den jeweiligen Akteur (und damit auch für seine verwendete Technologie) eingestuft. Im Extremfall, in dem die Standardabweichung $\sigma$ der maximalen absoluten Abweichung $c_{A B S}$ entspricht (d. h. die Differenz $c_{A B S^{-}} \sigma$ ist null), entfällt der Korrekturterm sogar komplett.

Berücksichtigt man nunmehr sowohl den Aspekt der Interdependenz der Standardisierungsentscheidungen als auch die Ermittlung der Informationskosten, so ergibt sich der Erwartungswert $E\left(S_{j}\right)$ der einsparbaren Kommunikationskosten von Akteur $j$ aus Sicht von Akteur $i$ wie folgt:

$$
E\left(S_{j}\right)=\sum_{\substack{k=1 \\ k \neq j}}^{M} \widetilde{c}_{j k} \cdot p_{i k}-K_{j}=\sum_{\substack{k=1 \\ k \neq j}}^{M}\left(c_{j i}-\frac{c_{A B S}-\sigma}{c_{A B S}} \cdot\left(c_{j i}-\bar{c}\right)\right) \cdot p_{i k}-K_{j}
$$

Dadurch lässt sich die Standardisierungswahrscheinlichkeit $p_{i j}$ des Akteurs $j$ ermitteln. Analog zu Akteur $j$ ermittelt Akteur $i$ auch für alle weiteren Akteure $k \in\{1, \ldots, M\}$ mit $k \neq j$ die Wahrscheinlichkeit $p_{i k}$. Somit ergibt sich ein System von $M$ Gleichungen, wobei in jeder Gleichung jeweils die $(M-1)$ Standardisierungswahrscheinlichkeiten der Akteure enthalten sind. Mit diesem Gleichungssystem sind nunmehr die Interdependenzen der Standardisierungsentscheidungen modellendogen und konsistent berücksichtigt.

Die Formulierung des Entscheidungsverhaltens auf Basis der Annahmen A.5* und A.6* hat insbesondere folgende wesentliche Vorteile im Vergleich zu den Arbeiten von Buxmann et al. (1999) und Weitzel et al. (2006):

1) Bei der Ermittlung der Standardisierungswahrscheinlichkeit für einen Akteur werden die Wahrscheinlichkeiten für die übrigen Akteure nicht mehr pauschal gleich eins gesetzt. Vielmehr wird die Interdependenz der Entscheidungen - was gerade den Gedanken der Netzeffekte charakterisiert - mittels eines Gleichungssystems modellendogen repräsentiert.

2) Für den nicht irrelevanten Fall, dass $c_{j i} \cdot(M-1)-K_{j}<0$ gilt, wird bisher mit $p_{i j}=0$ eine Knickstelle definiert (vgl. Ausdruck (3)), um für die Wahrscheinlichkeit $p_{i j}$ den Wertebereich $[0 ; 1]$ zu gewährleisten. Diese Knickstelle kann zu Verzerrungen bei der Berücksichtigung der Entscheidungen anderer Akteure führen. Dies wird mit Hilfe des Gleichungssystems vermieden, d. h. eine solche Knickstelle ist nicht mehr notwendig.

3) Bisher finden sich die erwarteten Einsparungen bei den Informationskosten aus Gründen der Normierung der Standardisierungswahrscheinlichkeit $p_{i j}$ auf $p_{i j} \leq 1$ auch im Nenner wieder (vgl. Ausdruck (3)). Das hat zur Folge, dass Wahrscheinlichkeiten $p_{i j}$ nahe eins nur dann auftreten, wenn die Standardisierungskosten $K_{j}$ im Vergleich zu den Einsparungen sehr klein sind. So wird selbst wenn die erwarteten Einsparungen bei den Informationskosten im Vergleich zu den Standardisierungskosten bspw. doppelt so groß sind, nur eine Wahrscheinlichkeit $p_{i j}$ von 0,5 ausgewiesen. Der neue Ansatz kommt ohne diese Art der Normierung aus.

Um das gestaltungsorientierte Entscheidungsverhalten sowie dessen Einfluss auf die Standardisierungslücke zu verdeutlichen, wird das Beispiel der sechs Institute weitergeführt.

Wiederum sind, wie oben, zunächst für jeden einzelnen Akteur $i$ (hier Institut $i$ ) die Wahrscheinlichkeiten $p_{i j}$ zu bilden. Betrachtet man beispielhaft erneut Institut 2, so ergeben sich folgende Werte für die Wahrscheinlichkeiten $p_{2 j}$ :

\begin{tabular}{|l|c|c|c|c|c|}
\hline & Institut 1 & Institut 3 & Institut 4 & Institut 5 & Institut 6 \\
\hline Wahrscheinlichkeit $p_{2_{j}}$ & 0,73 & 0,95 & 0,87 & 0,89 & 0,93 \\
\hline
\end{tabular}

Tab. 3: Ermittelte Wahrscheinlichkeiten aus Sicht von Institut 2 (neuer Ansatz) 
Auf dieser Basis ergeben sich aus Sicht von Institut 2 erwartete Einsparungen bei den Informationskosten in Höhe von $442.454 \mathrm{GE}$. Der Erwartungswert unter Berücksichtigung der Standardisierungskosten berechnet sich damit zu $E\left(S_{i}\right)=442.454 \mathrm{GE}-449.888 \mathrm{GE}=-7.434 \mathrm{GE}$. D. h. Institut 2 entscheidet auch hier, nicht zu standardisieren. Wenn die anderen Institute ebenfalls die Ergebnisse des neuen Ansatzes berücksichtigen, resultieren jeweils folgende erwartete Einsparungen bei den Kommunikationskosten $E\left(S_{i}\right)$ :

\begin{tabular}{|l|c|c|c|c|c|c|}
\hline & Institut 1 & Institut 2 & Institut 3 & Institut 4 & Institut 5 & Institut 6 \\
\hline $\begin{array}{l}\text { Erwartete } \\
\text { Einsparung } E\left(S_{i}\right)[\mathrm{GE}]\end{array}$ & -415.978 & -7.434 & 643.014 & 192.360 & 446.649 & 196.597 \\
\hline
\end{tabular}

Tab. 4: Erwartete Einsparungen $E\left(S_{i}\right)$ für die sechs Institute (neuer Ansatz)

Demnach standardisieren die Institute 3 bis 6, wodurch sich ex post folgende Einsparungen ergeben: $387.302 \mathrm{GE}$ (Institut 3), $63.834 \mathrm{GE}$ (Institut 4), 329.581 GE (Institut 5), -26.263 (Institut 6). Unter Berücksichtigung der theoretischen Einsparungen bei zentraler Entscheidung und vollständiger Information von 1.655.141 GE (s. o.) resultiert damit eine reduzierte Standardisierungslücke von nur noch 900.687 GE. Im Vergleich zum Ansatz von Buxmann et al. (1999) und Weitzel et al. (2006) entspricht dies einer Verringerung der Standardisierungslücke um annähernd 50\% (858.333 GE).

\section{Simulationsstudie zur Analyse der Standardisierungslücke}

Um zu untersuchen, in welchen Entscheidungssituationen und in welchem Umfang eine Standardisierungslücke auftritt bzw. reduziert werden kann, reicht das einfache Beispiel der X.500-Verzeichnisdienste nicht aus. Deshalb erfolgt im Weiteren eine tiefergehende Analyse. Dafür muss analog zu den Ansätzen von Buxmann (1996), Buxmann et al. (1999) und Weitzel et al. (2006) aus Gründen der Komplexität der Modellierung und um aussagekräftige Ergebnisse zu erhalten, auf Simulationsexperimente zurückgegriffen werden (vgl. hierzu aus methodologischer Sicht auch Bertrand/Fransoo 2002). So ist bspw. für einen Ausweis der Standardisierungslücke allein schon die Lösung des vergleichsweise einfachen Standardisierungsproblems bei zentraler Entscheidung und vollständiger Information nicht mehr in geschlossener Form sondern lediglich als Gleichungssystem (vgl. Domschke et al. 2002) darstellbar. Den mit einer Simulationsstudie einhergehenden Nachteilen (geringere analytische Transparenz, keine Darstellung der Ergebnisse in geschlossener Form etc.) steht dabei jedoch die Möglichkeit gegenüber, komplexere und dynamische Strukturen, simultane Parametervariationen sowie diskrete Entscheidungen untersuchen zu können (vgl. Weitzel et al. 2006; Wiese 1990).

Bei den Simulationsexperimenten finden jeweils die Parameterkonfigurationen aus (Buxmann et al. 1999; Weitzel et al. 2003a; Weitzel 2004; Weitzel et al. 2006) Verwendung ${ }^{8}$. Dies soll nicht nur Kontinuität gewährleisten. Vielmehr wird so eine konsistente und nachvollziehbare Gegenüberstellung und Analyse der Ergebnisse im Vergleich zu bestehenden Beiträgen möglich und der Kritik einer Beeinflussung der Ergebnisse durch selbst entwickelte Konfigurationen vorgebeugt. Zudem untersuchen diese Parameterkonfigurationen durch die Variation der Analysevariablen immer größere Definitionsbereiche, so dass keine Ergebnisse und Aussagen auf Basis einer einzelnen Partialsicht abgeleitet werden.

In der ersten Konfiguration von Buxmann et al. (1999) werden normalverteilte Informationskosten $c_{i j}$ mit Erwartungswert $E\left(c_{i j}\right)$ und konstanter Standardabweichung $\sigma\left(c_{i j}\right)=200$ zugrunde gelegt. Der Erwartungswert $E\left(c_{i j}\right)$ wird beginnend bei null kontinuierlich bis auf 1.700 erhöht (in Schritten zu je 50 Einheiten), wobei je Schritt 100 Simulationsläufe durchgeführt werden. 
Die Standardisierungskosten $K_{i}$ sind mit konstantem $E\left(K_{i}\right)=10.000$ und $\sigma\left(K_{i}\right)=1.000$ ebenfalls normalverteilt. Die Anzahl der Akteure ist mit $M=20$ fest und gegeben.

Eine graphische Illustration der Einsparungen im Netzwerk bei zentraler Entscheidung und vollständiger Information (,zentrale Lösung“) vs. den Einsparungen bei dezentraler Entscheidungsstruktur und vollständiger Information gemäß dem Entscheidungsverhalten bei Buxmann et al. (1999) und Weitzel et al. (2006) (,dezentrale Lösung“) liefert Abb. 7. Hierbei sind die variierten Erwartungswerte $E\left(c_{i j}\right)$ der Informationskosten auf der Abszisse sowie die Summe der ex post tatsächlich realisierten Einsparungen im gesamten Netzwerk auf der Ordinate abgetragen (als Durchschnitt aller 100 Simulationsläufe je $E\left(c_{i j}\right)$ ). Die Differenz zwischen den Einsparungen bei zentraler vs. bei dezentraler Lösung entspricht der jeweiligen Standardisierungslücke (vgl. Abschnitt 3.4), die zur besseren Darstellung als Fläche abgebildet ist.

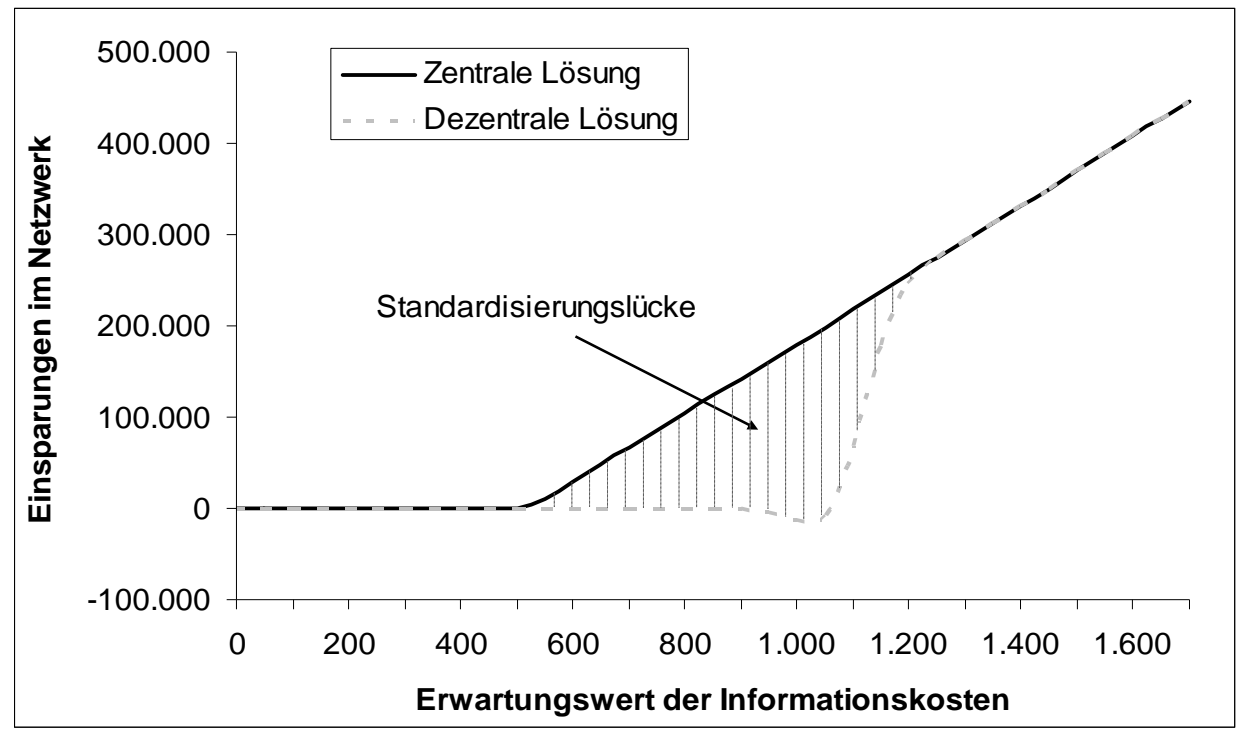

Abb. 7: Graphische Darstellung der Standardisierungslücke

Wie die Graphik verdeutlicht, werden bei der zentralen Lösung ab einem Erwartungswert von $E\left(c_{i j}\right)=500$ Einsparungen realisiert, die mit einem höheren Erwartungswert der Informationskosten $E\left(c_{i j}\right)$ ansteigen. Dagegen standardisiert bei der dezentralen Lösung im Bereich $E\left(c_{i j}\right) \in\left[0 ; 900\right.$ [ kein Akteur, was für $E\left(c_{i j}\right) \in[500 ; 900$ [ zu einer Standardisierungslücke führt. Diese setzt sich fort, da - wie bereits in (Buxmann et al. 1999) beschrieben - zwar ab einem Erwartungswert von $E\left(c_{i j}\right)=900$ auch bei dezentraler Lösung einzelne Akteure standardisieren, jedoch die durchschnittlichen, kumulierten Einsparungen im Bereich $E\left(c_{i j}\right) \in[900 ; 1.050] \mathrm{zu}-$ nächst sogar kleiner als null sind. D. h. aus der Entscheidung einiger Akteure für eine Standardisierung folgen ex post negative Einsparungen (Standardisierungskosten größer als die Summe der eingesparten Informationskosten). Grund dafür ist, dass bei dezentraler Lösung andere Akteure im Netzwerk zum Teil noch nicht, wie von den betroffenen Akteuren erwartet, ebenfalls standardisieren. Dadurch werden die erwarteten Einsparungen bei den Informationskosten ex post nicht im vollen Umfang tatsächlich realisiert (vgl. obiges Beispiel der sechs Institute). $\mathrm{Ab} E\left(c_{i j}\right)=1.100$ werden die kumulierten Einsparungen im Netzwerk bei dezentraler Lösung positiv. Diese steigen dann an, wobei ab einem Wert von $E\left(c_{i j}\right)=1.300$ die zentrale Lösung der dezentralen Entscheidung entspricht, d. h. es tritt keine Standardisierungslücke mehr auf.

In Abschnitt 3.4 wurden mit dem Problem fehlender Kompensation und dem Start-upProblem die beiden Ursachen für das Auftreten einer Standardisierungslücke diskutiert. Diese lassen sich im Simulationsexperiment analysieren. So tritt das Problem fehlender Kompensation im Intervall [500; 700] des Erwartungswerts der Informationskosten auf. Dies ist darauf zurück zuführen, dass hier für einzelne Akteure die Standardisierungskosten nicht durch die eigene Kosteneinsparung (bei zentraler Lösung) gedeckt werden. Das Problem fehlender 
Kompensation tritt bei $4.628(6,6 \%)$ von insgesamt 70.000 Standardisierungsentscheidungen der Akteure (35 Schritte 20 Akteure 100 Simulationsläufe) nur relativ selten auf. Dagegen ist das Start-up-Problem deutlich häufiger zu verzeichnen: Es tritt im Intervall [600;1.250] des Erwartungswerts der Informationskosten bei insgesamt 17.172 Entscheidungen (24,5\%) auf. Insofern würde sich eine Standardisierung für diese Akteure nur dann lohnen, falls andere Akteure ebenfalls standardisieren. Letztere verzichten jedoch aufgrund der unvollständigen Information auf die Einführung des Standards, obwohl dies für alle Akteure vorteilhaft wäre. Im Weiteren wird untersucht, inwieweit der gestaltungsorientierte Ansatz - im Folgenden mit DeStA (Dezentraler Standardisierungs-Ansatz) bezeichnet - dazu beitragen kann, die Standardisierungslücke zu reduzieren und Ineffizienzen zu vermeiden. Die Simulation mit DeStA wurde mit Hilfe einer Softwareimplementierung in Java 5.0 durchgeführt ${ }^{9}$. Da für die Berechnung der Gleichungssysteme auf numerische Optimierungsverfahren zurückzugreifen ist, wurden Routinen der Mathematiksoftware Mathematica ${ }^{\mathrm{TM}}$ eingebunden. Zur Gewährleistung der Realisierungsqualität und der Ergebnisrobustheit wurden mehrere numerische Optimierungsverfahren genutzt, um die Standardisierungsentscheidungen der Akteure zu ermitteln.

Eine Illustration der Ergebnisse liefert Abb. 8, die eine deutliche Reduzierung der Standardisierungslücke zeigt. Bei Verwendung von DeStA standardisieren bereits ab $E\left(c_{i j}\right)=500$ einzelne Akteure, dort jedoch noch nicht in gleichem Umfang wie bei der zentralen Lösung. Deswegen sind die kumulierten Einsparungen des Netzwerks ex post (im Durchschnitt über 100 Simulationsläufe) negativ. In diesen Fällen nehmen somit einzelne Akteure die Standardisierungskosten in Kauf und erzielen zunächst keine ausreichenden Einsparungen bei den Informationskosten. Bei den später dargestellten mehrperiodigen Betrachtungen ist allerdings festzustellen, dass sich dieser Effekt schnell nivelliert (andere Akteure ziehen bei der Einführung des Standards nach), so dass auch für diese Akteure in den nachfolgenden Perioden keine negativen Einsparungen mehr auftreten. Insofern ist der Effekt der negativen Einsparungen nur temporär, d. h. bei mehrperiodiger Betrachtung ergibt sich auch hier ex post ein positiver Kapitalwert der Standardisierung. Möchte man die (temporär) negativen Einsparungen dennoch vermeiden bzw. in ihrer Höhe reduzieren, kann bspw. ein risikoaverses Verhalten der Akteure angenommen und das Erwartungswertkalkül (vgl. Ausdruck (2)) um ein Risikomaß erweitert werden. Allerdings widerspricht dies Annahme A.4 $4_{\mathrm{DZ}}$ und steht der Vergleichbarkeit von DeStA mit bestehenden Ansätzen (z. B. Buxmann et al. 1999; Weitzel et al. 2006) entgegen. Deshalb wird im Weiteren die Annahme der Risikoneutralität beibehalten ${ }^{10}$.

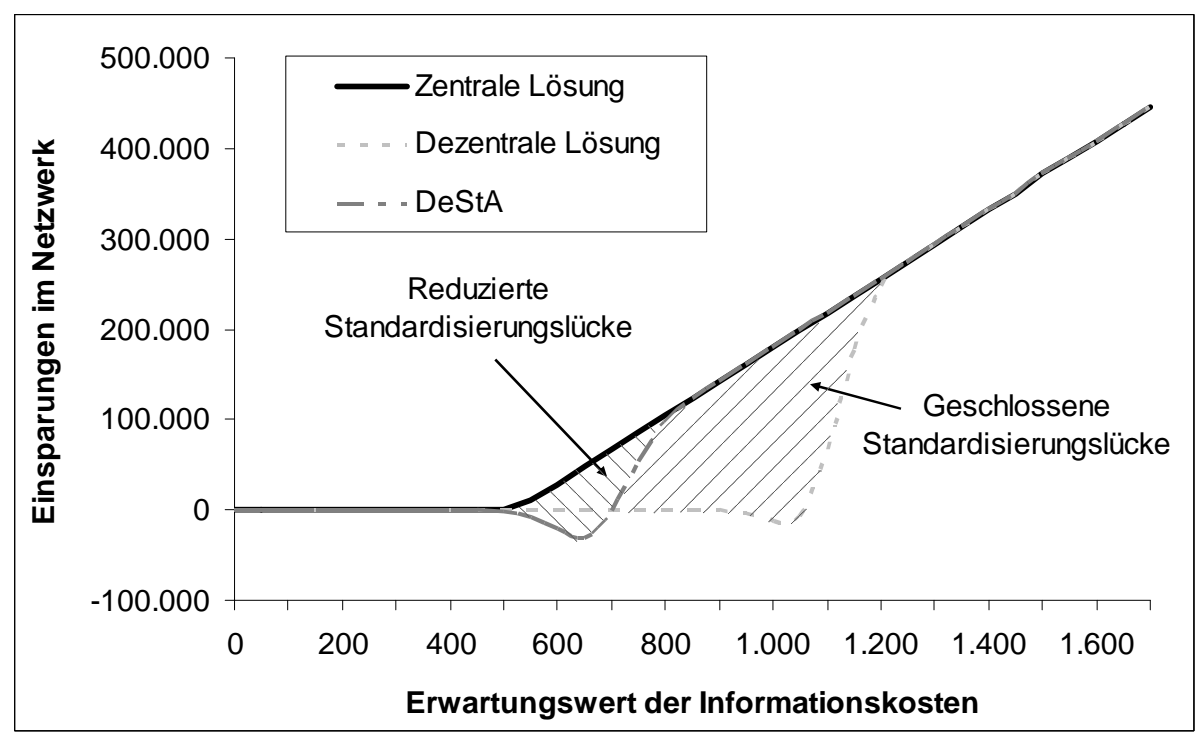

Abb. 8: Reduzierung der Standardisierungslücke bei systematisch variiertem Erwartungswert $E\left(c_{i j}\right)$ der Informationskosten

$\mathrm{Ab}$ einem Erwartungswert von $E\left(c_{i j}\right)=750$ werden dagegen positive kumulierte Einsparungen erzielt, die ab $E\left(c_{i j}\right)=950$ die volle Höhe an Einsparungen der zentralen Lösung erreichen. Vergleicht man die zentrale Lösung, die dezentrale Lösung gemäß dem Ansatz bei Buxmann 
et al. (1999) und Weitzel et al. (2006) sowie den neuen Ansatz DeStA, so lässt sich festhalten, dass sich im Simulationsexperiment die Ineffizienzen in Form der Standardisierungslücke wesentlich reduzieren. Da dieses Ergebnis jedoch auch von der Parameterkonfiguration abhängig ist, erfolgt im Weiteren eine Analyse bei systematischer Variation des Erwartungswerts $E\left(K_{i}\right)$ der Standardisierungskosten, der Standardabweichungen $\sigma\left(c_{i j}\right)$ für die Informationskosten sowie der Standardabweichungen $\sigma\left(K_{i}\right)$ für die Standardisierungskosten.

Aufbauend auf der Konfiguration in (Weitzel et al. 2003a) wird im zweiten Simulationsexperiment zuerst der Erwartungswert $E\left(K_{i}\right)$ der Standardisierungskosten beginnend mit null bis auf 23.000 in Schritten zu je 1.000 Einheiten erhöht, wobei je Schritt 100 Simulationsläufe erfolgen. Der Erwartungswert $E\left(c_{i j}\right)$ für die Informationskosten beträgt 1.000, die Standardabweichung $\sigma\left(c_{i j}\right)=200$. Beide werden, wie die Standardabweichung $\sigma\left(K_{i}\right)=1.000$ und die Anzahl der Akteure mit $M=20$, konstant gehalten ${ }^{11}$. Eine Darstellung der Ergebnisse liefert Abb. 9, bei der die variierten Erwartungswerte $E\left(K_{i}\right)$ auf der Abszisse sowie die kumulierten Einsparungen im Netzwerk auf der Ordinate abgetragen sind.

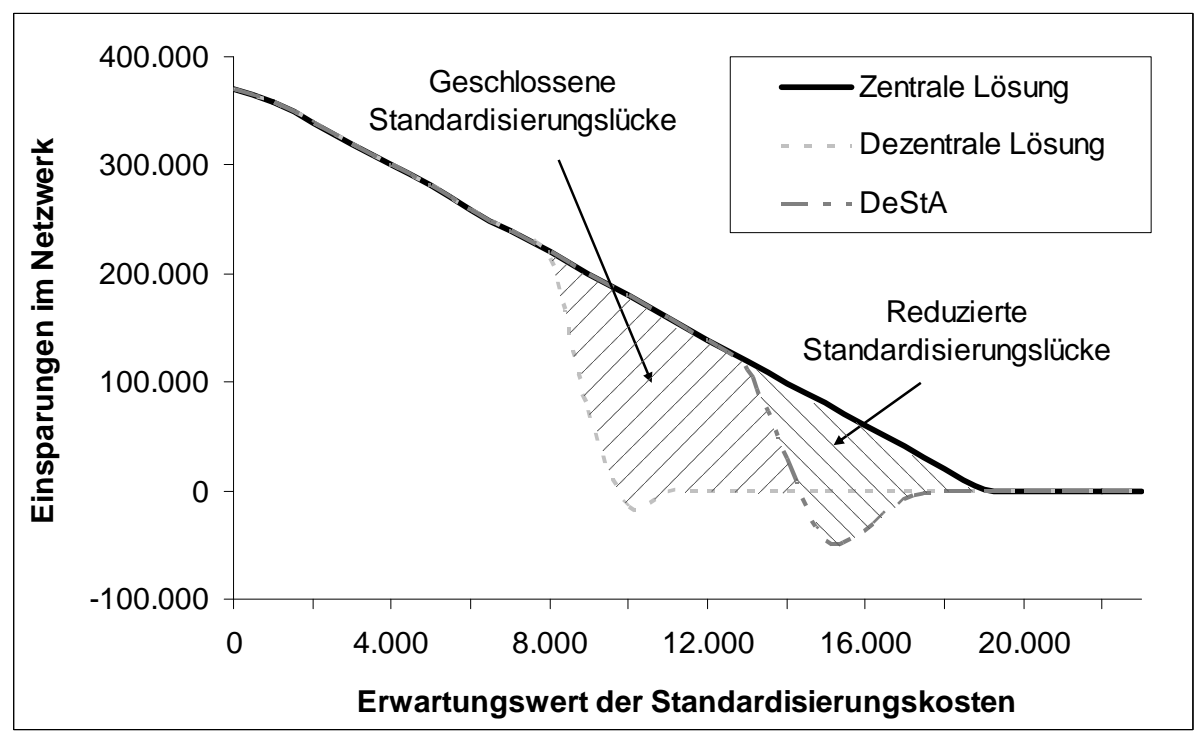

Abb. 9: Reduzierung der Standardisierungslücke bei systematisch variiertem Erwartungswert $E\left(K_{i}\right)$ der Standardisierungskosten

Die Graphik verdeutlicht, dass auch im zweiten Simulationsexperiment bei systematischer Variation des Erwartungswerts $E\left(K_{i}\right)$ der Standardisierungskosten DeStA erheblich zur Reduzierung der Standardisierungslücke beiträgt.

Schließlich wird im dritten Simulationsexperiment zusätzlich zur Variation des Erwartungswerts $E\left(K_{i}\right)$ der Standardisierungskosten im Bereich [3.000; 21.000] auch deren Standardabweichung $\sigma\left(K_{i}\right)$ systematisch variiert (vgl. Weitzel et al. 2003a). Die Standardabweichung $\sigma\left(K_{i}\right)$ der Standardisierungskosten wird dazu beginnend mit 500 bis auf 5.000 schrittweise erhöht (um je 500 Einheiten), wobei je Schritt 100 Simulationsläufe erfolgen. Der Erwartungswert $E\left(c_{i j}\right)$ für die Informationskosten beträgt 1.000, die Standardabweichung $\sigma\left(c_{i j}\right)=200$. Die Anzahl der Akteure $M$ beträgt 20. Eine Darstellung der Ergebnisse liefert Abb. 10, bei der die Standardabweichung $\sigma\left(K_{i}\right)$ und der Erwartungswert $E\left(K_{i}\right)$ der Standardisierungskosten auf den Abszissen sowie die Standardisierungslücke bei dezentraler Lösung (linke Seite) bzw. bei Verwendung des neuen Ansatzes (rechte Seite) auf der Ordinate abgetragen sind. Auch hier reduziert sich die Standardisierungslücke signifikant. Variiert man in einem weiteren Simulationsexperiment die Standardabweichung $\sigma\left(c_{i j}\right)$ für die Informationskosten, so resultieren analoge Ergebnisse. 

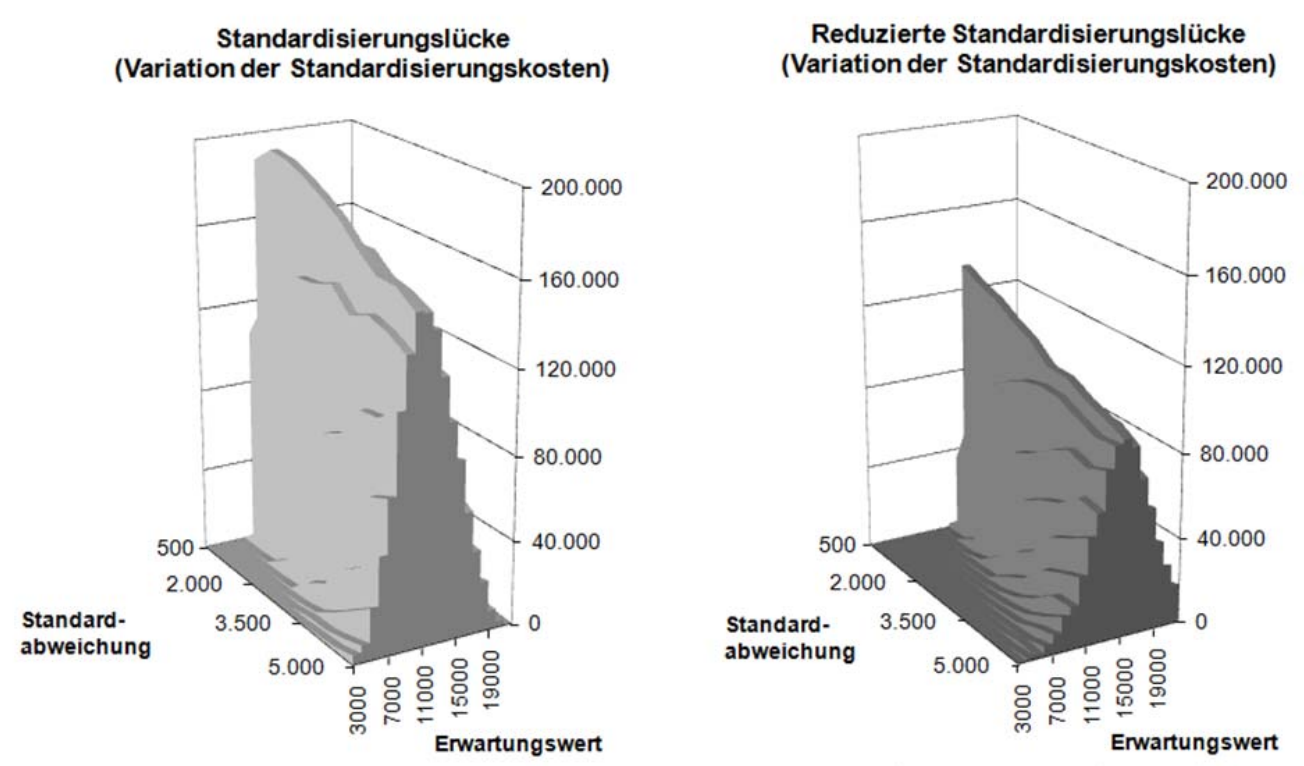

Abb. 10: Reduzierung der Standardisierungslücke bei einer systematischen Variation der Standardabweichung $\sigma\left(K_{i}\right)$ und des Erwartungswerts $E\left(K_{i}\right)$ der Standardisierungskosten

Neben diesen Simulationsexperimenten ist auch der Fall einer wiederholten Anwendung des obigen einperiodigen Entscheidungsmodells (nicht im Sinne eines dynamischen Optimierungsmodells) zu betrachten (vgl. Weitzel 2004). D. h. zu Beginn $t=1$ hat noch kein Akteur standardisiert (vgl. Annahme A.1), wohingegen zu Beginn der Perioden $t=2,3, \ldots, T$ jedem Akteur bekannt ist, welche Akteure bereits standardisiert haben und somit in Periode $t$ den Standard nutzen. Ist einem Akteur $i$ zu Beginn der Periode $t$ bekannt, dass ein anderer Akteur $j$ bereits standardisiert hat, so ist zur Berechnung des Erwartungswerts $E_{t}\left(S_{i}\right)$ der Periode $t$ die Wahrscheinlichkeit $p_{i j}$ auf eins zu setzen. Ein Akteur standardisiert dabei genau in derjenigen Periode $t$, in der für ihn erstmalig ein positiver Erwartungswert $E_{t}\left(S_{i}\right)$ der einsparbaren Kommunikationskosten resultiert.

Auf diese Weise wird für mehrere Perioden analysiert, in welchem Umfang sich eine Standardisierungslücke ergibt, inwieweit sich diese reduzieren lässt und welche Gleichgewichte (identische Anzahl standardisierender Akteure in den Perioden $t, t+1, \ldots, T-1, T$ ) sich in Netzwerken bei dezentraler Entscheidungsstruktur ergeben. Die Analyse erfolgt für einen Zeithorizont von zehn Perioden $(T=10)$. Als Basis dient beispielhaft die Parameterkonfiguration des ersten Simulationsexperiments (Variation des Erwartungswerts $E\left(c_{i j}\right)$ der Informationskosten). Die Ergebnisse sind in Abb. 11 dargestellt.
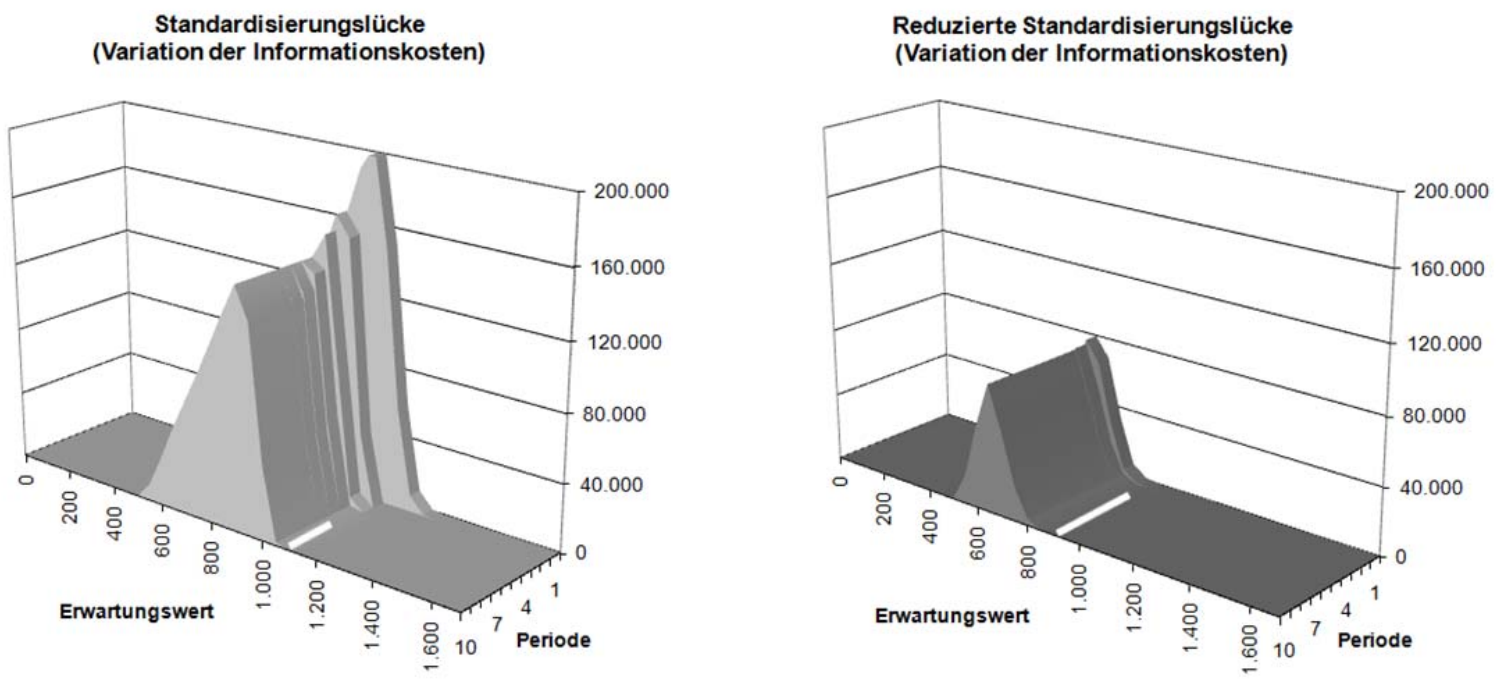

Abb. 11: Reduzierung der Standardisierungslücke bei systematisch variiertem Erwartungswert $E\left(c_{i j}\right)$ der Informationskosten und zehn Perioden $(T=10)$ 
Hier wird deutlich, dass durch Verwendung von DeStA bereits ab $T=2$ und $E\left(c_{i j}\right)=900$ die Standardisierungslücke vollständig geschlossen wird. Interessant ist zudem, dass bei Verwendung von DeStA die Gleichgewichtspfade insgesamt sehr kurz sind, d. h. bereits ab $T=4$ ist die Anzahl der Akteure, die den Standard nutzen, bei jedem Simulationslauf identisch.

Daneben wurde DeStA auch bei geänderter Netzwerktopologie analysiert. Dabei wurde wieder die Konfiguration des ersten Simulationsexperiments betreffend eine so genannte Random Topology (vgl. Weitzel et al. 2003b) zugrunde gelegt, bei der jeder Akteur $i$ mit einer festen, gegebenen Anzahl zufällig ermittelter Akteure $j$ keine Kommunikationsbeziehung unterhält (d. h. $c_{i j}=0$ ). Gerade für elektronische Märkte stellt dies laut Weitzel et al. (2003b) eine geeignete Konfiguration dar. Hier zeigen die Ergebnisse, dass auch bei einer verringerten Anzahl an Kommunikationspartnern (bspw. 18, 16, 14, 12 oder 10 Akteure) die Standardisierungslücke mit DeStA signifikant reduziert wird.

\section{Ergebnisse und Implikationen aus der Simulationsstudie}

Insgesamt lassen sich für den gestaltungsorientierten Ansatz DeStA zur Antizipation dezentraler Standardisierungsentscheidungen auf Basis der durchgeführten Simulationsexperimente folgende Ergebnisse und Implikationen festhalten:

1) [Spieltheoretische Fundierung des neuen Ansatzes] Im Vergleich zu einer rein entscheidungstheoretischen Modellierung der Akteure als (starre) Umweltzustände, werden bei DeStA die Interdependenz der Standardisierungsentscheidungen modellendogen im Ansatz repräsentiert und das resultierende Gleichungssystem gelöst. Damit erfolgt eine spieltheoretische Fundierung, da für die Ermittlung der Vorteilhaftigkeit einer Standardisierung aus Sicht eines Akteurs die Kalküle der jeweils anderen Akteure im Netzwerk wechselseitig eingehen und damit unmittelbar Berücksichtigung finden. Dass die Verwendung von DeStA vorteilhaft ist, zeigen neben den dargelegten Gründen am Ende von Abschnitt 4 auch die Simulationsexperimente. Zudem lässt sich anhand einer PayoffMatrix mit den ex post tatsächlich realisierten Einsparungen darstellen, dass die Verwendung von DeStA ein rationales Verhalten repräsentiert. Vergleicht man nämlich die Einsparungen der existierenden Strategien , standardisiere nie“, „standardisiere immer", „entscheide gemäß Buxmann et al. (1999) und Weitzel et al. (2006) “ (kurz: ,dezentrale Lösung“) und „entscheide gemäß des gestaltungsorientierten Ansatzes DeStA“ (kurz: „DeSt $A^{\text {“) }}$, so entspricht die zuletzt genannte Strategie der wechselseitig besten Antwort (Nash-Gleichgewicht). Die Pay-off Matrix in Abb. 12 zeigt dies für die Konfiguration des ersten Simulationsexperiments (vgl. Buxmann et al. 1999) ${ }^{12}$. Dabei repräsentieren die Zeilen den Aktionsraum für einen einzelnen Akteur (sei dies o. B. d. A. Akteur 1) mit den jeweils durchschnittlich realisierbaren Einsparungen und die einzelnen Spalten den Aktionsraum der anderen Akteure (seien dies o. B. d. A. die Akteure 2-20). Ein Akteur wählt somit seine Strategie unter Berücksichtigung der Strategien der anderen Akteure und der jeweils durchschnittlich realisierbaren Einsparungen. Diese Strategiewahl führt jeder Akteur in gleicher Weise durch, wobei die Matrix den Fall zeigt, dass die anderen Akteure zunächst jeweils die gleiche Strategie verfolgen. 


\begin{tabular}{|c|c|c|c|c|c|c|c|c|c|c|}
\hline & \multicolumn{8}{|c|}{ Strategie der Akteure 2-20 } \\
\hline & & & \multicolumn{2}{|c|}{ 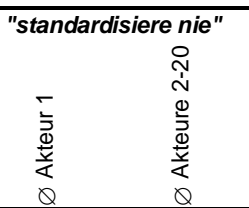 } & \multicolumn{2}{|c|}{ 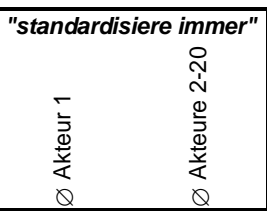 } & \multicolumn{2}{|c|}{ 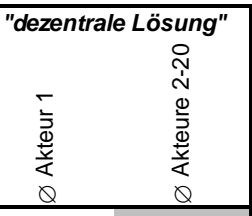 } & 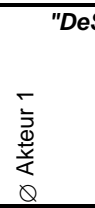 & 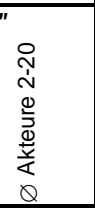 \\
\hline \multirow{8}{*}{ 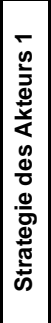 } & \multirow{2}{*}{$\begin{array}{c}\text { "standardisiere } \\
\text { nie" }\end{array}$} & $\varnothing$ Akteur 1 & \multirow[t]{2}{*}{0} & & \multirow[t]{2}{*}{0} & & \multicolumn{2}{|c|}{0} & \multirow{2}{*}{0} & \\
\hline & & $\varnothing$ Akteure 2-20 & & 0 & & 5.626 & & 5.309 & & 6.714 \\
\hline & \multirow{2}{*}{\begin{tabular}{|c|} 
"standardisiere \\
immer"
\end{tabular}} & $\varnothing$ Akteur 1 & -10.001 & & 6.492 & & \multirow[t]{2}{*}{-107} & & \multirow[t]{2}{*}{3.687} & \\
\hline & & $\varnothing$ Akteure 2-20 & & 0 & & 6.492 & & 5.831 & & 7.437 \\
\hline & \multirow{2}{*}{$\begin{array}{l}\text { "dezentrale } \\
\text { Lösung" }\end{array}$} & $\varnothing$ Akteur 1 & -3.684 & & 6.176 & & \multirow[t]{2}{*}{5.810} & & \multirow[t]{2}{*}{6.176} & \\
\hline & & $\varnothing$ Akteure 2-20 & & 0 & & 6.146 & & 5.810 & & 7.235 \\
\hline & \multirow{2}{*}{ "DeStA" } & $\varnothing$ Akteur 1 & -6.007 & & 7.707 & & 3.888 & & \multirow[t]{2}{*}{7.422} & \\
\hline & & $\varnothing$ Akteure 2-20 & & 0 & & 6.348 & & 5.831 & & 7.422 \\
\hline
\end{tabular}

Abb. 12: Pay-off Matrix

Hier wird deutlich, dass es für einen Akteur nicht vorteilhaft ist, von der Strategie „DeS-

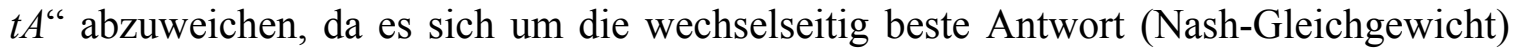
handelt (vgl. ähnlich auch Diekmann/Voss 2004). Da ein Akteur die Entscheidung der anderen Akteure nicht beeinflussen kann (bspw. andere Akteure zwingen, eine bestimmte Strategie zu wählen) und er diese nicht kennt, ist es aus seiner Sicht rational, dass kein anderer Akteur von der Strategie „DeStA“ abweicht, da er sich dadurch keinen Vorteil verschaffen kann. Die höheren durchschnittlich realisierbaren Einsparungen des Akteurs 1 (bei Wahl von „DeStA“) falls alle anderen Akteure die Strategie ,standardisiere immer" verfolgen sind dadurch begründet, dass eine grundsätzliche Standardisierung aller anderen Akteure bei positiven Netzeffekten offensichtlich nur Vorteile für den Akteur 1 mit sich bringt. Allerdings kann dieser nicht beeinflussen, dass alle anderen Akteure tatsächlich immer standardisieren, so dass dies kein Gleichgewicht darstellt, zumal die durchschnittlich realisierbaren Einsparungen der anderen Akteure bei der Strategie ,standardisiere immer" deutlich geringer als bei Wahl der Strategie „DeStA“ ausfallen. Insofern ist es für die übrigen Akteure nicht rational, die Strategie ,standardisiere immer" zu wählen. Gleiches gilt für die Strategiekombination, in welcher der Akteur 1 ,standardisiere immer" und die übrigen Akteure „DeStA“ wählen, da dies für den Akteur 1 nachteilig ist. Wenn aber ein Abweichen von der Strategie „DeStA“ für einen einzelnen Akteur nicht rational ist und keine Abstimmungen zwischen den Akteuren erfolgen, ist es auch nicht rational, überhaupt darauf zu spekulieren, dass ein oder mehrere Akteure davon abweichen. Sollte ein Akteur dennoch darauf spekulieren, dass einzelne (irrationale) Akteure auf eine andere Strategie setzen, so bleibt die beste Antwort trotzdem - so zeigen weitere Analysen ${ }^{13}$ - jeweils die Strategie „DeStA “. Insofern ist es für Akteure rational, die Interdependenz der Standardisierungsentscheidungen modellendogen bei der Entscheidungsfindung einzubeziehen und den gestaltungsorientierten Ansatz zu verwenden.

2) [Implikationen mit Bezug zum Start-up-Problem] Das Start-up-Problem beruht auf einer wechselseitigen Blockade der Akteure. Will jeder Akteur die Vorteilhaftigkeit einer Standardisierung unter Sicherheit bewerten, so muss er die Entscheidung der anderen Akteure kennen. Keiner möchte das ,überproportionale Risiko des ersten Technologienutzers eingehen" (vgl. Weitzel/König 2003). Dies kann dazu führen, dass Akteure mit ihrer Entscheidung ,aufeinander warten“ und es, obwohl eine Standardisierung ökonomisch vorteilhaft ist, nicht zur besten Lösung für die Akteure kommt. Das Start-up-Problem resultiert dabei ausschließlich aus der unvollständigen Information der Akteure und erfordert im Prinzip keine Kompensation (vgl. Abschnitt 3.4). Die Simulationsstudie zeigt Folgendes: Bei einer systematischen Variation des Erwartungswerts $E\left(c_{i j}\right)$ der Informationskosten wird durch die Verwendung des Ansatzes DeStA die Anzahl der Start-upProbleme wesentlich reduziert (schon bei einer Betrachtungsperiode um über 90\%). Gleiches gilt bei einer systematischen Variation der Standardabweichung $\sigma\left(K_{i}\right)$ und des Erwartungswerts $E\left(K_{i}\right)$ der Standardisierungskosten (bei einer Betrachtungsperiode hier um 
über 83\%). Dies verdeutlicht, dass durch die modellendogene Repräsentation der Interdependenz der Standardisierungsentscheidungen zur Lösung des Start-up-Problems erheblich beigetragen wird ${ }^{14}$. Interessant ist zudem, dass dies auch bei einer mehrperiodigen Betrachtung im Sinne der oben erläuterten wiederholten Anwendung des einperiodigen Modells sowie bei Veränderungen der Netzwerktopologie gilt. Daneben ist die Zeitdauer bis zum Eintritt des Gleichgewichts (Stabilität der Lösung) bei einer mehrperiodigen Betrachtung kurz, d. h. die Standardisierungslücke wird im Zeitverlauf sehr schnell reduziert. Die mit einer Unterstandardisierung verbundenen negativen ökonomischen Folgen lassen sich demnach stark reduzieren, ohne dass auf komplexe Koordinationsmechanismen wie eine virtuelle Instanz (vgl. Weitzel et al. 2001), Versicherungslösungen (vgl. König/Weitzel 2003) oder eine zentrale Architekturabteilung (vgl. Heinrich et al. 2006) zurückgegriffen werden muss. Auch die (oftmals unrealistische) Prämisse eines vollständigen Austauschs korrekter Information zwischen nicht-opportunistischen Akteuren, die den Koordinationsmechanismen dezentraler Abstimmung oftmals zugrunde liegt (z. B. schwarzes Brett mit verbindlichen Ankündigungen oder Verhandlungen im Sinne eines Bandwagon-Prozesses), ist hier nicht notwendig.

Neben diesen generellen Implikationen soll kurz auf eine spezifische Simulationskonfiguration eingegangen werden. So wird von Weitzel et al. (2003a) bei systematischer Variation der Standardabweichung der Standardisierungskosten richtigerweise gefolgert, dass , ,increasing deviation goes along with an increasing efficiency of decentralized coordination compared to the centralized solution". D. h. gerade bei einer kleineren Standardabweichung der Standardisierungskosten ist die Standardisierungslücke im Vergleich zu einer höheren Standardabweichung größer. Netzwerke, in denen die Akteure über eine ähnliche Kostenstruktur verfügen (homogene Netzwerke), stellen somit aus ökonomischer Sicht ein besonderes Problem dar. Speziell hier kann DeStA jedoch gemäß den Ergebnissen des Simulationsexperiments zu einer Lösung beitragen, da die Anzahl der Start-upProbleme bei homogenen Netzwerken hier wesentlich stärker reduziert wird.

3) [Implikationen mit Bezug zum Problem fehlender Kompensation] Neben dem Startup-Problem basiert die Standardisierungslücke auch darauf, dass eine Standardisierung einzelner Akteure zwar nicht aus deren dezentraler Sicht, jedoch aus Netzwerksicht vorteilhaft ist. Fehlen in diesem Fall entsprechende Mechanismen (z. B. Kompensationszahlungen), kommt es zum Problem fehlender Kompensation und einer Unterstandardisierung (vgl. Abschnitt 3.4). Diesbezüglich ist Folgendes festzuhalten: Zwar kann der neue Ansatz DeStA hier ebenfalls unterstützen. Jedoch ist zu beachten, dass DeStA das dezentrale Kalkül eines Akteurs zugrunde liegt und nicht die zentrale Lösung aus Netzwerksicht. Vor diesem Hintergrund soll DeStA gerade keine Empfehlungen für dezentrale Entscheidungen geben, die zwar letztlich zur zentralen Lösung führen, jedoch für einzelne Akteure aus deren dezentraler Sicht nachteilig sind. Diese Akteure würden ansonsten die zentrale Lösung „mit realisieren“, ohne jedoch eine entsprechende Kompensation für ihre Standardisierungskosten zu erhalten (Problem fehlender Kompensation). Allerdings soll DeStA in diesem Fall zukünftig zu einer Lösung beitragen. So zielen viele bestehende Ansätze auf eine Koordination der Kompensationszahlungen durch eine zentrale Instanz ab. Wie in Abschnitt 3.5 diskutiert, ist die Einführung solcher zentraler Koordinationsmechanismen jedoch aus diversen Gründen (z. B. Durchsetzungsproblem bei Kompensationszahlungen, Verrechnungsproblem bei den Koordinationskosten, Problem der Einforderung vollständiger und richtiger Informationen von allen Akteuren) durchaus kritisch. Insofern erscheint es aussichtsreich, DeStA für die Abschätzung einer notwendigen bzw. hinreichenden Kompensation (aus Sicht eines jeden Akteurs) weiter zu entwickeln. Hier gilt es jeweils die Sicht der anderen Akteure im Netzwerk einzunehmen, um zu untersuchen, welche Akteure potenziell eine Kompensation in welcher Höhe benötigen bzw. leisten können, um zur Realisierung der zentralen Lösung beizutragen. Der Erwartungswert der einsparbaren Kommunikationskosten eines Akteurs (vgl. Ausdruck (4)) ist dafür ein geeigneter Ausgangspunkt. Er bildet aus Sicht eines Akteurs nicht nur ab, ob andere 
Akteure im Netzwerk potenziell standardisieren, sondern liefert mit dem Abstand zur Indifferenz (Erwartungswert gleich null) zugleich einen Anhaltspunkt, inwieweit diese eine Kompensation benötigen bzw. leisten können. Hieraus ergeben sich $u$. a. folgende zukünftige Forschungsfragen: In welchen Fällen gibt es ein Einigungsintervall für Kompensationen? Wie sieht dieses aus (ggf. auch hinsichtlich einer „fairen“ Kompensation) und innerhalb welcher Grenzen kann dem opportunistischen Verhalten eines Akteurs vorgebeugt werden (spieltheoretischer Aspekt)?

\section{Zusammenfassung}

Im Beitrag wurden das Standardisierungsproblem bei dezentraler Entscheidungsstruktur und unvollständiger Information fokussiert und ein neuer, gestaltungsorientierter Ansatz zur Antizipation dezentraler Standardisierungsentscheidungen und der zu erwartenden Netzeffekte entwickelt. Dieser soll die einzelnen Akteure im Netzwerk bei der Entscheidung über die Einführung eines Standards unterstützen. Wichtige Ergebnisse sind:

- Bisherige Ansätze zielen primär darauf ab, das Auftreten theoretischer Phänomene, wie z. B der Standardisierungslücke, zu erklären. Im Vergleich dazu wurde im Beitrag ein gestaltungsorientierter Ansatz entwickelt, welcher der konkreten Entscheidungssituation eines Unternehmens bei der Einführung von Kommunikationsstandards Rechnung trägt.

- Die Beiträge von Buxmann et al. (1999) und Weitzel et al. (2006) bilden den maßgeblichen Ausgangspunkt für die Entwicklung des neuen Ansatzes. Im Gegensatz zu diesen Beiträgen steht jedoch bei der Modellierung des dezentralen Entscheidungsverhaltens im Mittelpunkt, die Interdependenz der Standardisierungsentscheidungen modellendogen zu repräsentieren und das resultierende Gleichungssystem zu lösen.

- Die modellendogene Berücksichtigung der Interdependenz der Standardisierungsentscheidungen gewährleistet die spieltheoretische Fundierung des neuen Ansatzes. Dabei lässt sich aufzeigen, dass dessen Verwendung im Vergleich zu alternativen Strategien rationales Verhalten repräsentiert (vgl. Abschnitt 6). Daneben verdeutlicht die Simulationsstudie, dass der gestaltungsorientierte Ansatz wesentlich zur Reduzierung der Standardisierungslücke und zur Verbesserung ineffizienter, dezentraler Entscheidungen beiträgt.

- Zudem zeigt die Simulationsstudie auf, dass das Start-up-Problem mit Hilfe des gestaltungsorientierten Ansatzes wesentlich reduziert werden kann. Somit lassen sich die negativen ökonomischen Folgen einer Unterstandardisierung in Netzwerken stark verringern, ohne dass auf Koordinationsmechanismen zurückgegriffen werden muss, deren Umsetzung in der Realität oft kritisch ist. Das Problem fehlender Kompensation kann und soll der entwickelte Ansatz wegen des zugrunde liegenden dezentralen Kalküls dagegen bewusst nicht direkt lösen. Trotzdem kann der vorgestellte Ansatz hier durchaus eine geeignete Grundlage für zukünftige Arbeiten bilden, die dieses Problem adressieren.

Abschließend sind auch einige kritische Punkte zu diskutieren, die weiteren Forschungsbedarf darstellen. So soll ein gestaltungsorientierter Ansatz Akteuren, deren Standardisierung zwar aus Netzwerksicht (zentrale Lösung), nicht jedoch aus deren dezentraler Sicht vorteilhaft ist (Problem fehlender Kompensation), die Einführung des Standards nicht empfehlen. Ohne entsprechende Kompensation würden diese Akteure benachteiligt. Insofern ist kritisch zu hinterfragen, ob die zentrale Lösung als Referenzgröße zur Beurteilung des dezentralen Entscheidungsverhaltens überhaupt geeignet ist. König und Weitzel (2003) sind diesbezüglich ähnlich zu interpretieren, wenn sie schreiben: „, Natürlich ist die Interpretation der aggregierten Nutzen CE ("coordination efficiency") nicht unproblematisch in dezentralen Netzwerken, da dort der Fokus auf Individualentscheidungen liegt. Ziel ist die Konstruktion einer Vergleichsgröße möglicher Entscheidungsgüte bei der Entscheidung über die Vernetzung von Akteuren ". Von Weitzel (2004) wird hier das so genannte ex post-Kriterium zur Beurteilung der Güte des dezentralen Entscheidungsverhaltens vorgeschlagen. Dazu werden zwei Arten von Fehlent- 
scheidungen definiert: Entweder der Akteur standardisiert und bereut diese Entscheidung, da dadurch ex post negative Einsparungen realisiert werden. Oder er sieht von einer Standardisierung ab, obwohl dies ex post vorteilhaft gewesen wäre. Einerseits berücksichtigt dieses Kriterium, dass dezentral optimale Entscheidungen getroffen werden sollen, d. h. die zentrale Lösung wird sinnvollerweise nicht mehr fokussiert. Andererseits wird die Referenzgröße, gegen welche die Entscheidungsgüte (vgl. Weitzel et al. 2006) zu beurteilen ist, durch das Entscheidungsverhalten (oder die gewählte Strategie) selbst direkt beeinflusst. Offensichtlich wird die Problematik, wenn man analysiert, welche Güte so mit der trivialen Strategie ,standardisiere nie" erzielt werden kann. Wird diese von allen Akteuren im Netzwerk gewählt, so standardisiert im Ergebnis kein Akteur, was zugleich als Referenz für die Beurteilung der Entscheidungsgüte selbst dient. Wenn jedoch kein Akteur im Netzwerk standardisiert, so ist auch die Entscheidung jedes einzelnen Akteurs nicht zu standardisieren ex post richtig. Somit treten nach dem ex post-Kriterium keine Fehlentscheidungen auf, obwohl aus dieser Strategie i. d. R. offensichtlich erhebliche Ineffizienzen (wegen der Unterstandardisierung) resultieren. Insofern ist eine Referenzgröße zu definieren, mit der beurteilt werden kann, in welchem Ausmaß ein Akteur selbst bei unvollständiger Information so entscheidet wie er sich im Falle vollständiger Information entschieden hätte. Folglich könnte die Entscheidungsgüte an den dezentralen Standardisierungsentscheidungen gemessen werden, die auf Basis vollständiger Information zustande kommt. Gerade diese Lücke zwischen vollständiger und unvollständiger Information soll adressiert werden, um Fehlentscheidungen aus dezentraler Sicht zu vermeiden. Deshalb erscheint es durchaus aussichtsreich, als Referenzgröße zukünftig die optimale Lösung bei dezentraler Entscheidungsstruktur und vollständiger Information weiter zu analysieren und zu verwenden.

Darüber hinaus ist auch die bisherige Simulationsstudie weiterzuführen bzw. kritisch zu hinterfragen. Hierbei wurde auf die Parameterkonfigurationen bspw. von Buxmann et al. (1999) und Weitzel (2004) zurückgegriffen, um Kontinuität und Vergleichbarkeit mit bestehenden Analysen zu gewährleisten. Obwohl die Simulationsstudie bereits unterschiedliche Experimente mit jeweils größeren Definitionsbereichen für die Modellparameter untersucht und damit keine Aussagen aufgrund einer einzelnen Partialsicht abgeleitet wurden, ist künftig trotzdem noch genauer zu untersuchen, welche Auswirkungen die verwendeten Parameterkonfigurationen auf die Ergebnisse und Implikationen haben. Zur Verdeutlichung mag hier die folgende in (Weitzel et al. 2003a) zum Problem fehlender Kompensation getroffene Aussage dienen: ,Moreover, if particular agents would suffer individually but contribute to aggregate efficiency [...] a compensation plan needs to be established since the option model would not per se solve the start-up problem by its pure existence but also some side payments would have to be made. But according to the simulations, these cases are quite rare". Legt man hier jedoch bspw. die Parameterkonfiguration des ersten Simulationsexperiments zugrunde, so tritt das Problem fehlender Kompensation im Bereich [0;1.700], in dem der Erwartungswert der Informationskosten variiert wird, keineswegs immer selten auf. Vielmehr ist das Problem im Bereich $[500 ; 700]$ bei fast jedem zweiten Akteur und im Bereich [900; 1.100] dagegen nicht zu verzeichnen. Da die Häufigkeit des Auftretens natürlich nicht nur Einfluss auf die Relevanz des Problems, sondern ebenso auf den Einsatz geeigneter Mechanismen hat, gilt es künftig, mögliche Abhängigkeiten der Ergebnisse und Implikationen von der Parameterkonfigurationen im Detail zu untersuchen.

Neben den kritischen Punkten und den Erweiterungsmöglichkeiten sind künftig auch mehrere, alternative Standards zu berücksichtigen. Ein wertvoller Beitrag hierzu wird von Weitzel et al. (2006) geliefert. Darauf basierend ist zu untersuchen, welche Ergebnisse und Implikationen sich hier aus der Verwendung des neuen, gestaltungsorientierten Ansatzes ergeben. Zudem stellt die Ermittlung der Kosten weiteren Forschungsbedarf dar (bspw. die Eliminierung von Ausreißern bei der Antizipation der unbekannten Informationskosten). Dies wurde im Beitrag nicht detailliert beleuchtet. Insofern gilt es, mögliche Alternativen und deren Güte zu untersuchen. Der vorgestellte Ansatz bietet dafür jeweils eine Grundlage. 


\section{Anmerkungen}

1 Dies muss nicht bedeuten, dass im Falle einer Standardisierung keine Kosten mehr für die Kommunikation anfallen. Die Kosten $c_{i j}$ können auch als Differenz zwischen den Informationskosten vor $\left(c_{i j}^{v}\right)$ und nach $\left(c_{i j}^{n}\right)$ der Standardisierung interpretiert werden, d. h. sie entsprechen dann mit $c_{i j}^{v}-c_{i j}^{n}=c_{i j}$ den infolge der Standardisierung auf der zugehörigen Kante einsparbaren Informationskosten.

${ }^{2}$ Die Annahme, dass in der Ausgangssituation noch kein Akteur standardisiert hat, kann problemlos relaxiert werden, wie später bei der Betrachtung mehrerer Perioden deutlich wird.

3 Auch diese Annahme kann problemlos geändert werden, wenn allen Akteuren bekannt ist, welche Akteure keine Kommunikationsbeziehungen unterhalten. Ist dies nicht bekannt, so hat eine Abschätzung, bspw. auf Basis des durchschnittlichen Vermaschungsgrads, zu erfolgen.

${ }^{4}$ Für den Fall, dass die Kosten der Kommunikation (realistischerweise) bei der Nutzung des Standards nicht komplett entfallen, ist wie in Endnote 1 beschrieben zu verfahren.

${ }^{5}$ Die Kostengrößen $c_{i j}$ und $K_{i}$ können auch barwertig interpretiert werden, d. h. es fallen in zukünftigen Perioden Informations- und Standardisierungskosten an, die auf den Entscheidungszeitpunkt diskontiert werden.

${ }^{6}$ Falls die Informationskosten dennoch zum Teil bekannt sind, können diese ohne Weiteres zusätzlich berücksichtigt werden.

${ }^{7}$ Die nachstehende Modellformulierung wurde aus Gründen der Nachvollziehbarkeit gewählt. Bei der Simulationsstudie wurde im Hinblick auf die Rechenzeit auf eine LP-Relaxation zurückgegriffen (vgl. Domschke et al. 2002; Domschke/Wagner 2005).

${ }^{8}$ In den Beiträgen (Weitzel et al. 2006) und (Weitzel et al. 2003a) werden Simulationsexperimente mit zum Teil anderen Konfigurationen (bspw. mit $M=35$ Akteuren) durchgeführt. Bei Verwendung dieser Konfigurationen ändern sich die grundsätzlichen Ergebnisse jedoch nicht.

9 Für die Simulationsstudie wurde der Term (5) beispielhaft wie folgt instanziiert: Um einen Wertebereich von $(-1 ; 1)$ zu erhalten, erfolgte eine Multiplikation mit dem Faktor $2 / \pi$. Anschließend wurde $\pi / 4$ addiert, um die notwendige Verschiebung nach oben zu erreichen. Dies wiederum erforderte eine weitere Stauchung der Arcustangens-Funktion durch Multiplikation mit dem Faktor (1- $\pi / 4)$, um den Wertebereich der Standardisierungswahrscheinlichkeiten $p_{i j}$ nach oben durch 1 zu beschränken.

${ }^{10}$ Für eine detaillierte Diskussion zu den negativen Einsparungen im Bereich [500; 700] für den Erwartungswert der Informationskosten und wie diese überwunden werden können siehe Anhang 2.

${ }^{11}$ Werden bei dieser Simulationskonfiguration Werte für die Standardisierungskosten $K_{i}<0$ gezogen, so wird die zufällige Ziehung wiederholt. Alternativ können die Standardisierungskosten für diesen Fall auch auf den Wert null gesetzt werden, was jedoch eine Verzerrung nach sich zieht, da nur dieser einzelne Wert dann - in Bezug zur Verteilungsannahme - häufiger auftritt.

${ }^{12}$ Bei anderen Parameterkonfigurationen ergeben sich analoge Ergebnisse.

${ }^{13}$ Die Ergebnisse einiger ausgewählter Analysen sind in Anhang 3 dargestellt.

${ }^{14}$ Diese Aussage beruht darauf, dass sich bei einem Verzicht der Antizipation der unbekannten Informationskosten anhand des Ausdrucks (6) die obigen Ergebnisse nur unwesentlich ändern. 


\section{Literatur}

Besen SM, Farrell J (1994) Choosing how to compete: Strategies and Tactics in Standardization. J Econ Perspect 8(2):117-131

Bertrand JWM, Fransoo JC (2002) Modelling and Simulation: Operations Management Research Methodologies using quantitative Modeling. Int J Oper Prod Manag 22(2):241264

Buxmann P (1996) Standardisierung betrieblicher Informationssysteme. Gabler-Verlag, Wiesbaden

Buxmann P, Weitzel T, König W (1999) Auswirkung alternativer Koordinationsmechanismen auf die Auswahl von Kommunikationsstandards. Z Betriebswirtsch 69(2):133-155

Diekmann A, Voss T (2004) Die Theorie rationalen Handelns. Stand und Perspektiven. In: Diekmann A, Voss T (Hrsg) Rational-Choice-Theorie in den Sozialwissenschaften. Anwendungen und Probleme. Oldenbourg, München, S 13-29

Domschke W, Mayer G, Wagner B (2002) Effiziente Modellierung von Entscheidungsproblemen: Das Beispiel des Standardisierungsproblems. Z Betriebswirtsch 72(8):847-863

Domschke W, Wagner B (2005) Models and Methods for Standardization Problems. Eur J Oper Res 162(3):713-726

Farrell J (1987) Cheap Talk, Coordination and Entry. Rand J Econ 18(1):34-39

Farrell J, Saloner G (1985) Standardization, Compatibility, and Innovation. Rand J Econ 16(1):70-83

Farrell J, Saloner G (1986) Installed Base and Compatibility: Innovation, Product Preannouncements, and Predation. Am Econ Rev 76(5):940-955

Farrell J, Saloner G (1988) Coordination through Committees and Markets. Rand J Econ 19(2):235-252

Heinrich B, Klier M, Bewernik M (2006) Unternehmensweite Anwendungsintegration Zentrale Anreizsetzung zur Realisierung von Netzwerkeffekten bei dezentralen Entscheidungsstrukturen. Wirtschaftsinformatik 48(3):158-168

Katz ML, Shapiro C (1985) Network Externalities, Competition, and Compatibility. Am Econ Rev 75(3):424-440

König W, Weitzel T (2003) Netzeffekte im E-Business. In: Uhr W, Esswein W, Schoop E (Hrsg) Wirtschaftsinformatik 2003 - Medien - Märkte - Mobilität, Dresden, September 2003, 1. Band. Physica, Heidelberg, S 9-33

Liebowitz SJ, Margolis SE (1995) Are Network Externalities a new Source of Market Failure? Res Law Econ 17:1-22

Matutes C, Regibeau P (1988) Mix and Match: Product Compatibility without Network Externalites. Rand J Econ 19(2):221-234

Meredith JR, Raturi A, Amoako-Gyampah K, Kaplan B (1989) Alternative Research Paradigms in Operations. J Oper Manag 8(4):297-326

Rapoport A (2001) N-Person Game Theory: Concepts and Applications. Dover Publications, New York

Varian H (1994) A Solution to the Problem of Externalities when Agents are well-informed. Am Econ Rev 84(5):1278-1293

Weitzel T (2003) A Network ROI. In: Proceedings of the MISQ Academic Workshop on ICT Standardization, ICIS 2003, Seattle WA 2003, S 62-79

Weitzel T (2004) Economics of Standards in Information Networks. Physica-Verlag, Heidelberg

Weitzel T, Beimborn D, König W (2003a) Coordination in Networks: An economic Equilibrium Analysis. Inform Syst E Bus Manag 1(2):189-211 
Weitzel T, Beimborn D, König W (2006) A unified economic Model of Standard Diffusion: The Impact of Standardization Cost, Network Effects, and Network Topology. MIS Quarterly 30(Special Issue):489-514

Weitzel T, König W (2003) Computational Economics als wirtschaftsinformatischer Beitrag zu einer interdisziplinären Netzwerktheorie. Wirtschaftsinformatik 45(5):497-502

Weitzel T, Son S, König W (2001) Infrastrukturentscheidungen in vernetzten Unternehmen: Eine Wirtschaftlichkeitsanalyse am Beispiel von X.500 Directory Services. Wirtschaftsinformatik 43(4):371-381

Weitzel T, Wendt O, von Westarp F, König W (2003b) Network Effects and Diffusion Theory - Extending economic Network Analysis. Int J IT Stand Stand Res 1(2):1-21

Wendt O, von Westarp F, König W (2000) Diffusionsprozesse in Märkten für Netzeffektgüter

- Determinanten, Simulationsmodell und Marktklassifikation. Wirtschaftsinformatik 42(5):422-433

Wiese H (1990) Netzeffekte und Kompatibilität - Eine theoretische und simulationsgeleitete Analyse zur Absatzpolitik für Netzeffekt-Güter. Poeschel, Stuttgart

\title{
Introducing Communication Standards in Corporate Networks - a Normative Approach for a Decentralized Decision Structure
}

\begin{abstract}
Standards and technologies (e.g. in the area of web services) that are currently discussed in research and practice will strongly change IT-based communication between organizations. However, the decision to standardize is accompanied by the risk of introducing a standard that is not adopted by other organizations afterwards. Hence, the interdependencies among the standardization decisions due to positive network effects result in a coordination problem, which is referred to as "the standardization problem". If the standardization decisions are taken by autonomous actors (e.g. legally independent organizations) that do not know the standardization decisions of the other actors in the network, this standardization problem arises for a decentralized decision structure and incomplete information. Thus, actors have to decide under uncertainty on whether to standardize or not. To avoid resulting inefficiencies and wrong decisions, we propose a formal, normative approach. This approach allows an actor within the network to anticipate the standardization decisions of the other actors and the associated network effects. Central to our approach are the consideration of interdependencies among the standardization decisions and the solution of the resulting system of equations.
\end{abstract}

Keywords: Communication Standards, Network Effects, Standardization Problem, Decision Support, Decentralized Decision Structure 


\section{Anhang 1: Begründung der verwendeten Arcustangens-Funktion}

Die Anforderungen an eine Funktion zur Abbildung der Standardisierungswahrscheinlichkeiten $p_{i j}$ der Akteure $j$ auf Basis des Erwartungswerts $E\left(S_{j}\right)$ der einsparbaren Kommunikationskosten dieser Akteure im Falle einer Standardisierung lassen sich formal wie folgt festhalten:

F.1 [Definitionsbereich] Die Funktion soll auf $\mathbb{R}$ definiert sein, da die erwarteten Einsparungen Werte aus $\mathbb{R}$ annehmen können.

F.2 [Wertebereich] Die Funktion soll die erwarteten Einsparungen auf einen Wert aus dem Intervall $[0 ; 1]$ abbilden, um eine Interpretation als Wahrscheinlichkeit zu ermöglichen.

F.3 [Monotonie] Die Funktion soll streng monoton steigend sein, sodass sich eine höhere Einsparung in einer höheren Standardisierungswahrscheinlichkeit widerspiegelt.

Um die Standardisierungswahrscheinlichkeiten der anderen Akteure zu ermitteln und dabei die Interdependenz der Entscheidungen zu berücksichtigen, ist es nötig, dass für jeden Akteur ein Gleichungssystem mit $M$ Gleichungen gelöst wird. Dabei enthält jede Gleichung jeweils die $(M-1)$ Funktionen für die Standardisierungswahrscheinlichkeiten der anderen Akteure. Daraus ergeben sich zudem folgende Forderungen an die Funktion:

F.4 [Differenzierbarkeit] Die Funktion soll stetig differenzierbar sein, um die (numerischen) Verfahren zur Lösung der Gleichungssysteme nicht zu beeinträchtigen (Sicherstellung der Konvergenz).

F.5 [Darstellung] Die Funktion soll geschlossen darstellbar sein, um ebenfalls die Verfahren zur Lösung der Gleichungssysteme nicht zu beeinträchtigen.

Aus diesen Forderungen lassen sich folgende Charakteristika eines Funktionstyps $F(x)$ ableiten, auf dessen Basis die Abbildung der Standardisierungswahrscheinlichkeiten $p_{i j}$ erfolgen kann:

1) [Beschränktheit] Aus dem geforderten Wertebereich (F.2) folgt, dass der Funktionstyp $F(x)$ sowohl nach oben als auch nach unten beschränkt sein muss. Eine Beschränkung auf Werte aus dem Intervall $[0 ; 1]$ kann durch Stauchung und Verschiebung der Funktion erfolgen.

2) [Grenzverhalten] Aus der geforderten Monotonie (F.3), dem Wertebereich (F.2) und dem Definitionsbereich (F.1) der Funktion folgt, dass der Funktionstyp $F(x)$ sowohl für $x \rightarrow-\infty$ als auch für $x \rightarrow+\infty$ feste Grenzwerte aufweisen muss. Eine Beschränkung auf Werte aus dem Intervall $[0 ; 1]$ kann ebenfalls durch Stauchung und Verschiebung der Funktion vorgenommen werden.

3) [Krümmung] Aus der geforderten Monotonie (F.3) und dem Wertebereich (F.2) der Funktion folgt, dass ein geeigneter Funktionstyp $F(x)$ mindestens einen Wendepunkt besitzt.

Die Arcustangens-Funktion weist die geforderten Charakteristika auf, sodass auf deren Basis die Abbildung $p_{i j}=f\left(\operatorname{ArcTan}\left[\frac{E\left(S_{j}\right)}{K_{j}}\right]\right)$ definiert werden kann. Im Detail gilt Folgendes: Der Arcustangens ArcTan $[x]$ ist eine stetig differenzierbare Funktion (F.4), die geschlossen darstellbar (F.5) und auf ganz $\mathbb{R}$ definiert ist (F.1). Die Ableitung des Arcustangens ist mit $\frac{\partial \operatorname{ArcTan}[x]}{\partial x}=\frac{1}{1+x^{2}}$ für alle $x \in \mathbb{R}$ echt positiv, was einen streng monoton wachsenden Verlauf der Funktion zur Folge hat (F.3). Dabei nimmt der Arcustangens Werte aus dem Intervall $\left(-\frac{\pi}{2} ; \frac{\pi}{2}\right)$ an, ist somit sowohl nach oben als auch nach unten beschränkt ([Beschränktheit]) und weist mit $-\frac{\pi}{2}$ und $\frac{\pi}{2}$ feste Grenzwerte auf ([Grenzverhalten]). Durch einfache Stauchung und Verschiebung kann dabei eine Abbildung mit Werten aus dem Intervall $(0 ; 1)$ erreicht werden (F.2). Zudem besitzt ArcTan[x] bei $x=0$ den einzigen Wendepunkt, da hier die zweite Ablei- 
tung mit $\frac{\partial \operatorname{ArcTan}[x]}{\partial^{2} x}=-\frac{2 \cdot x}{\left(1+x^{2}\right)^{2}}$ den Wert null annimmt und der Term zugleich an dieser Stelle das Vorzeichen wechselt ([Krümmung]).

Neben der Arcustangens-Funktion erfüllen bspw. auch einige Verteilungsfunktionen von stetigen Zufallsvariablen (z. B. Normalverteilung, Extremwertverteilung und Students tVerteilung) die Forderungen. Obwohl diese Funktionen ebenfalls verwendbar sind (und eine Verwendung nicht zu grundsätzlichen anderen Ergebnissen führen würde), wäre eine Reihe größerer Probleme damit verbunden:

1) Die Verwendung einer Verteilungsfunktion wäre deswegen irreführend, da eine Interpretation der Funktion im wahrscheinlichkeitstheoretischen Sinne nicht möglich ist. Hierbei wären Fragen zu beantworten, wie: Welche Zufallsvariable wird betrachtet? Welche Verteilungsparameter liegen vor? Wie ist in diesem Zusammenhang der Wert der Funktion zu interpretieren? Diese Fragen können im vorliegenden Kontext nicht beantwortet werden (es existiert keine Zufallsvariable) oder nur, falls Annahmen bspw. bzgl. der Verteilung und der Verteilungsparameter getroffen werden, die jedoch ihrerseits nicht begründbar sind. Insgesamt würde die Auswahl demnach lediglich aufgrund der funktionalen Eigenschaften erfolgen, was jedoch eine Fehlinterpretation zur Folge haben könnte.

2) Die Verteilungsfunktionen sind zum Teil (z. B. Verteilungsfunktion der Normalverteilung) nicht geschlossen darstellbar, was u. a. Probleme bei der Durchführung der Verfahren zur Lösung der Gleichungssysteme schafft.

3) Die Verwendung einer Verteilungsfunktion könnte Zusammenhänge zwischen der Simulation der Kostengrößen im Netzwerk (Verwendung einer Normalverteilung) und dem Ansatz vermuten lassen, was für die Akzeptanz der Simulationskonfiguration und der Qualität der Ergebnisse wenig förderlich wäre.

Daher wurde im Beitrag auf die Arcustangens-Funktion zurückgegriffen. 


\section{Anhang 2: Diskussion der negativen Einsparungen im Bereich [500; 700] für den Erwar- tungswert der Informationskosten}

Abb. 8 illustriert neben den Einsparungen im Netzwerk bei zentraler und dezentraler Lösung auch die Einsparungen im Netzwerk, falls der neue Ansatz Verwendung findet. Hierbei wird einerseits deutlich, dass die Standardisierungslücke deutlich reduziert werden kann. Andererseits treten im Bereich [500; 700] für den Erwartungswert der Informationskosten negative Einsparungen auf, die kritisch zu diskutieren sind. Die negativen Einsparungen sind (analog zur bestehenden dezentralen Lösung) darin begründet, dass bei Verwendung von DeStA bereits einzelne Akteure bereits standardisieren, andere Akteure im Netzwerk dies jedoch noch nicht tun, obwohl dies ex ante von den erstgenannten Akteuren so erwartet wird. Deswegen sind die kumulierten Einsparungen im Netzwerk ex post noch negativ. D. h. in diesen Fällen tätigen einzelne Akteure Anfangsinvestitionen in die Einführung des Standards und erzielen zunächst (in der betrachteten Periode) keine ausreichenden Einsparungen bei den Informationskosten. Hierzu ist Folgendes anzumerken:

1) Die für einzelne Akteure ex post negativen Einsparungen in der betrachteten Periode nivellieren sich bei mehrperiodigen Betrachtungen i. d. R. sehr schnell, da in den Folgeperioden andere Akteure mit der Einführung des Standards nachziehen (dies verdeutlichen die mehrperiodigen Simulationsexperimente). Insofern ist der Effekt der negativen Einsparungen nur temporär und wird von den positiven Einsparungen in den Folgeperioden ausgeglichen, so dass sich insgesamt auch für die ersten Adoptoren ein ex post positiver Kapitalwert der Standardisierung ergibt. Dagegen erfolgt bei Verwendung der bestehenden dezentralen Lösung für diese Parameterkonstellationen grundsätzlich keine Einführung des Standards, weswegen auch in späteren Perioden keine positiven Einsparungen erzielt werden. Folglich sind bei einer mehrperiodigen Betrachtung die Akteure (auch die, die zunächst kurzfristig negative Einsparungen erleiden) bei dieser Parameterkonstellation im Vergleich zur bestehenden dezentralen Lösung nicht schlechter gestellt.

2) Möchte man die (temporär) negativen Einsparungen dennoch vermeiden oder in ihrer Höhe reduzieren, könnte bspw. methodisch wie folgt verfahren werden: Man verändert die Annahme der Risikoneutralität (vgl. Annahme A.4 Dz: „Er [Ein Akteur] standardisiert genau dann, wenn dies für ihn einen positiven Erwartungswert $E\left(S_{i}\right)$ der einsparbaren Kommunikationskosten zur Folge hat (Risikoneutralität)") und führt für die Akteure ein risikoaverses Verhalten ein. Für die Modellierung des dezentralen Entscheidungsverhaltens hat dies zur Folge, dass das reine Erwartungswertkalkül (vgl. Ausdruck (2)) um eine Risikokomponente zu erweitern ist, die einem risikoaversen Verhalten Rechnung trägt. Zu bewerkstelligen ist dies bspw. durch Einführung eines Risikoaversionsparameters $a$ (ArrowPratt-Maß) in Verbindung mit der Standardabweichung $\sigma$ der einsparbaren Kommunikationskosten als Risikomaß. Alternativ könnten (in Abhängigkeit der Verteilung der einsparbaren Kommunikationskosten) natürlich auch andere Risikomaße, wie z. B. DownsideRisikomaße, genutzt werden. Ein Akteur entscheidet demnach nicht mehr rein auf Basis des Erwartungswerts $\mu=E\left(S_{i}\right)$ der einsparbaren Kommunikationskosten, sondern auf Basis des Nutzenwerts, der zugleich die Risikokomponente beinhaltet. Abb. 13 ergänzt Abb. 8 exemplarisch um eine solche Risikobetrachtung und zeigt für verschiedene Ausprägungen des Risikoaversionsparameters $a$ die jeweils resultierende Standardisierungslücke. 


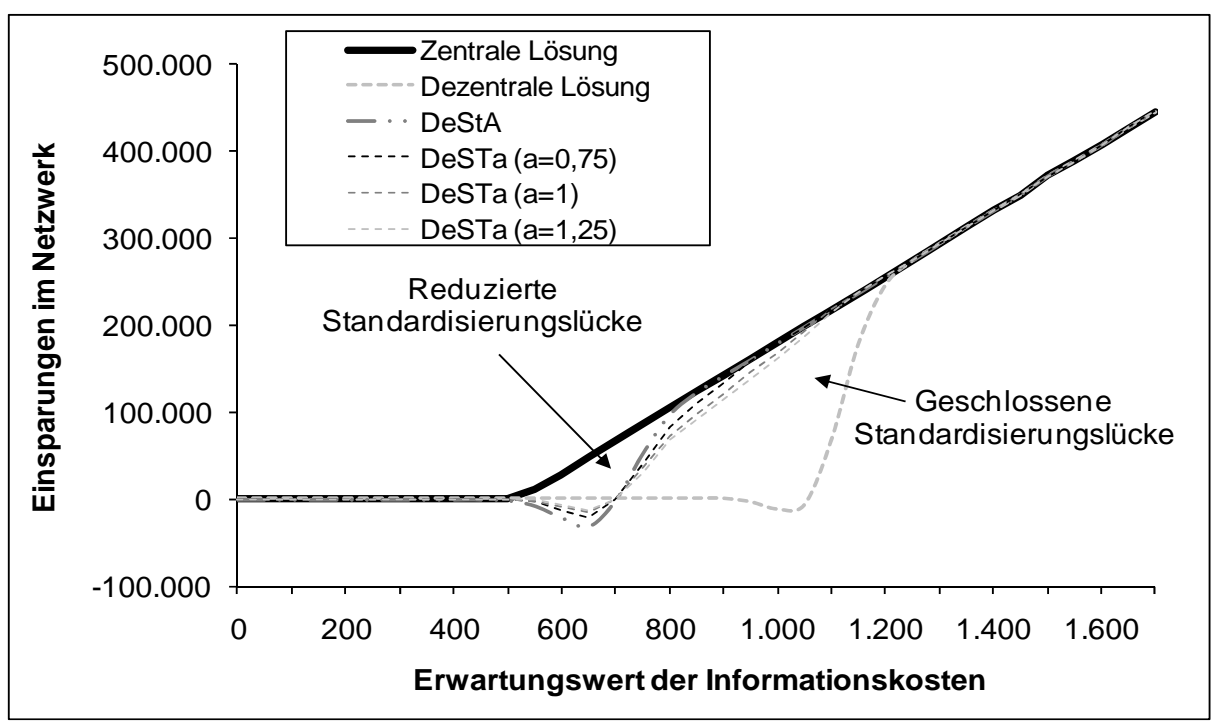

Abb. 13: Reduzierung der Standardisierungslücke bei Berücksichtigung einer Risikokomponente

Hier wird deutlich, dass bei einer höheren Risikoaversion der Umfang der negativen Einsparungen nachvollziehbarerweise immer mehr verringert wird. Für $a=1,25$ sind die negativen Einsparungen sogar betragsmäßig bereits durchweg geringer als bei der dezentralen Lösung. Gleichzeitig geht mit einer höheren Risikoaversion auch eine geringere Bereitschaft einher, den Standard einzuführen. Folglich wird die Standardisierungslücke in einem etwas geringeren Ausmaß geschlossen. Trotzdem ist die Standardisierungslücke, wie Abb. 13 illustriert, nach wie vor deutlich geringer als bei der dezentralen Lösung. 


\section{Anhang 3: Ergänzende spieltheoretische Untersuchungen}

Im Beitrag wurde gezeigt, dass die Verwendung des neuen, gestaltungsorientierten Ansatzes DeStA im Vergleich zu alternativen Strategien rationales Verhalten repräsentiert. Vor diesem Hintergrund ist es, wie bereits erläutert, auch nicht rational darauf zu spekulieren, dass ein oder mehrere Akteure auf eine andere Strategie setzen. Trotzdem wurde in einer Reihe weiterer Analysen die durchaus interessante Fragestellung untersucht, welche Konsequenzen es hätte, wenn manche Akteure trotzdem ein anderes Entscheidungsverhalten wählen und sich damit nicht rational verhalten würden. Die Ergebnisse einer ausgewählten Analyse werden im Folgenden dargestellt.

In Abb. 12 ist die Pay-off Matrix abgebildet, die sich auf Basis des ersten in Abschnitt 5 untersuchten Simulationsexperiments von Buxmann et al. (1999) ergibt, falls alle anderen Akteure im Netzwerk eine einheitliche Strategie verfolgen. Nun wird diese Analyse dahingehend modifiziert, dass es nunmehr Akteure gibt, die von der dominanten Strategie „DeStA“ abweichen und stattdessen bspw. das bestehende dezentrale Entscheidungsverhalten (,dezentrale Lösung") wählen. Das Ergebnis ist in Abb. 14 dargestellt.

Dabei besitzt ein Akteur (in den verschiedenen Zeilen) weiterhin den gesamten betrachteten Aktionsraum. Dagegen wird jetzt in den einzelnen Spalten betrachtet, was passiert, falls ein, zwei, drei, vier oder fünf Akteure nicht auf den neuen Ansatz setzen, sondern das bestehende dezentrale Entscheidungsverhalten wählen. Dabei zeigt sich, dass diese Akteure geringere Einsparungen realisieren, wobei sich die Strategiewahl der anderen Akteure nicht ändert. D. h. selbst wenn einzelne Akteure nicht das ermittelte Nash-Gleichgewicht anstreben, ist es immer noch rational für die anderen Akteure, den neuen Ansatz zu verwenden, um die jeweils höchsten Einsparungen zu realisieren. Die nicht rational agierenden Akteure würden dagegen niedrigere Einsparungen realisieren, was diese Strategiewahl ökonomisch zweifelhaft erscheinen lässt. Zugleich würde sich die Standardisierungslücke als Resultat der einzelnen dezentralen Entscheidungen in diesen Fällen entsprechend vergrößern.

\begin{tabular}{|c|c|c|c|c|c|c|c|c|c|c|c|c|c|c|c|c|c|}
\hline & \multicolumn{15}{|c|}{ Strategien der Akteure 2-20 } \\
\hline & & & \multicolumn{3}{|c|}{$\begin{array}{l}\text { 5x"dezentrale Lösung"/ } \\
14 x^{\prime \prime D e S t A " ~}\end{array}$} & \multicolumn{3}{|c|}{$\begin{array}{c}\text { 4x"dezentrale Lösung"| } \\
\text { 15x"DeStA" }\end{array}$} & \multicolumn{3}{|c|}{$\begin{array}{c}3 x " d e z e n t r a l e \text { Lösung" / } \\
16 x " D e S t A "\end{array}$} & \multicolumn{3}{|c|}{$\begin{array}{c}\text { 2x"dezentrale Lösung" } \\
17 x^{\prime \prime D e S t A " ~}\end{array}$} & \multicolumn{3}{|c|}{$\begin{array}{c}\text { 1x"dezentrale Lösung"/ } \\
18 x " D e S t A "\end{array}$} \\
\hline & & & 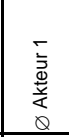 & 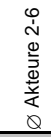 & 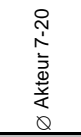 & 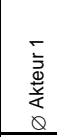 & 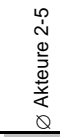 & 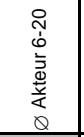 & 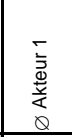 & 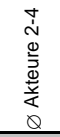 & 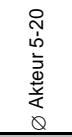 & 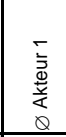 & 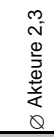 & 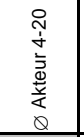 & 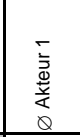 & 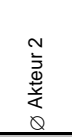 & 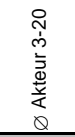 \\
\hline \multirow{12}{*}{ 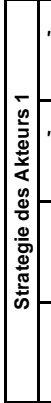 } & & $\varnothing$ Akteur 1 & ( & & & & & & 0 & & & ( & & & 0 & & \\
\hline & $\begin{array}{c}\text { standardislere } \\
\text { nie" }\end{array}$ & $\varnothing$ Akteure "dez. Lösung" & & 5.68 & & & 5.70 & & & 5.612 & & & 5.61 & & & 5.591 & \\
\hline & & $\varnothing$ Akteure "DeStA" & & & 5.987 & & & 5.963 & & & 5.962 & & & 6.140 & & & 6.115 \\
\hline & & $\varnothing$ Akteur 1 & 2.92 & & & 2.92 & & & 2.925 & & & 3.12 & & & 3.124 & & \\
\hline & $\begin{array}{l}\text { "standardisiere } \\
\text { immer" }\end{array}$ & $\varnothing$ Akteure "dez. Lösung" & & 6.24 & & & 6.27 & & & 6.132 & & & 6.13 & & & 6.107 & \\
\hline & & $\varnothing$ Akteure "DeStA" & & & 6.711 & & & 6.674 & & & 6.674 & & & 6.852 & & & 6.817 \\
\hline & & $\varnothing$ Akteur 1 & 6.17 & & & 6.18 & & & 6.109 & & & 6.11 & & & 6.108 & & \\
\hline & $\begin{array}{l}\text { 'dezentrale } \\
\text { Lösung" }\end{array}$ & $\varnothing$ Akteure "dez. Lösung" & & 6.17 & & & 6.18 & & & $6.10 s$ & & & 6.11 & & & 6.108 & \\
\hline & & $\varnothing$ Akteure "DeStA" & & & 6.507 & & & 6.482 & & & 6.481 & & & 6.659 & & & 6.633 \\
\hline & & $\varnothing$ Akteur 1 & 6.79 & & & 6.81 & & & 6.883 & & & 6.90 & & & 6.904 & & \\
\hline & "DeStA" & $\varnothing$ Akteure "dez. Lösung" & & 6.19 & & & 6.17 & & & 6.13 & & & 6.13 & & & 6.107 & \\
\hline & & $\varnothing$ Akteure "DeStA" & & & 6.792 & & & 6.813 & & & 6.883 & & & 6.904 & & & 6.904 \\
\hline
\end{tabular}

Abb. 14: Modifizierte Pay-off Matrix

Vergleichbare Ergebnisse treten auf, wenn einzelne Akteure die Strategien „standardisiere immer" oder ,standardisiere nie“" wählen. Demzufolge lässt sich festhalten, dass es für jeden Akteur rational ist, das Nash-Gleichgewicht und damit die Strategie „DeStA“ zu wählen. Dies gilt selbst unter der Annahme, dass einzelne Akteure nicht rational handeln und auf andere Strategien setzen. 